\title{
Investigation of Hydrodynamic Dispersion and Intra-pore Turbulence Effects in Porous Media
}

\author{
Nima Fallah Jouybari ${ }^{1}$ - T. Staffan Lundström ${ }^{1}$ · J. Gunnar I. Hellström ${ }^{1}$
}

Received: 13 July 2019 / Accepted: 6 November 2019 / Published online: 16 November 2019

(c) The Author(s) 2019

\begin{abstract}
The aim of the present paper is to evaluate and compare the pore level hydrodynamic dispersion and effects of turbulence during flow in porous media. In order to compute these quantities, large eddy simulations of turbulent flow in five unit cells comprised of spherical particles are performed and the results are averaged over the cells. Visualizations of vortical structures reveal that the size of the turbulence structures is of the size of the pores. Investigations furthermore yield that volume-averaged values of the hydrodynamic dispersion are of the same order as the Reynolds stress within the pores. It is also shown that the effect of intra-pore turbulence and hydrodynamic dispersion on the redistribution of macroscopic momentum within the porous medium is negligible compared to Forchheimer term. A discussion is provided on the accuracy of the eddy viscosity hypothesis in the modeling of the volume-averaged intra-pore Reynolds stresses. Finally, the effect of variation in the pore-scale geometry on the turbulence structures and averaged values of hydrodynamic dispersion and Reynolds stress is investigated.
\end{abstract}

Keywords Turbulence $\cdot$ Porous media $\cdot$ LES $\cdot$ Hydrodynamic dispersion $\cdot$ Reynolds stress

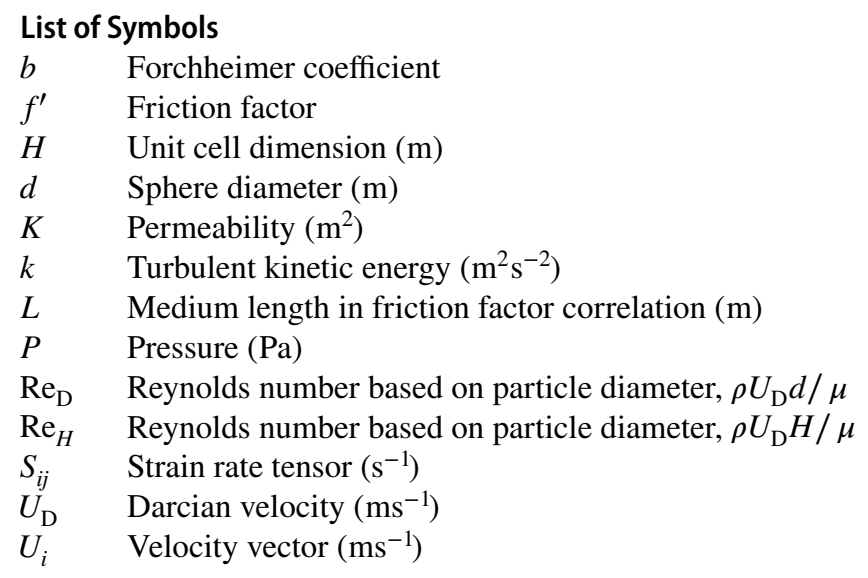

Nima Fallah Jouybari

nima.fallahjoybari@1tu.se

1 Division of Fluid Mechanics, Luleå University of Technology, 97187 Luleå, Sweden 


\section{$U, V \quad$ Velocity components $\left(\mathrm{ms}^{-1}\right)$ \\ $x, y, z \quad$ Cartesian coordinates $(\mathrm{m})$}

\section{Greek Symbols}

$\varphi \quad$ Porosity

$\Delta \quad$ Filter length

$\rho \quad$ Fluid density $\left(\mathrm{kg} \mathrm{m}^{-3}\right)$

$\mu \quad$ Fluid dynamic viscosity $\left(\mathrm{kg} \mathrm{m}^{-1} \mathrm{~s}^{-1}\right)$

$\mu_{t \varphi} \quad$ Turbulent viscosity in porous media $\left(\mathrm{kg} \mathrm{m}^{-1} \mathrm{~s}^{-1}\right)$

$v \quad$ Fluid kinematic viscosity $\left(\mathrm{m}^{2} \mathrm{~s}^{-1}\right)$

$v_{\text {SGS }} \quad$ SGS kinematic eddy viscosity

$\tau_{i j}^{r} \quad$ SGS stress tensor

\section{Special Characters}

$\phi \quad$ General variable

$\phi^{\prime} \quad$ Fluctuation from time average of $\phi$

$\langle\phi\rangle^{f} \quad$ Intrinsic average

$\bar{\phi} \quad$ Time average of $\phi$ in double decomposition or filtered $\phi$ in LES equations

$\tilde{\phi} \quad$ Fluctuation from volume average of $\phi$

\section{Introduction}

High velocity flows in porous media take place in industrial and natural applications such as cooling of electronic components, solid matrix heat exchangers, enhanced oil recovery, thermal insulation and flow over vegetation (Jouybari et al. 2015). This has motivated researchers to study turbulence effects in porous media, in more detail than before, the latest years (De Lemos 2009; Vafai 2015). To this end, experimental observation and categorization of different flow regimes in porous media have been undertaken in many studies (Dybbs and Edwards 1984; Seguin et al. 1998; Khayamyan et al. 2017a, b; Larsson et al. 2018). It is observed that the flow in porous media is fully turbulent at a pore-based Reynolds number, $\operatorname{Re}_{\mathrm{D}}=\rho U_{\mathrm{D}} d / \mu$, of a few hundred. To exemplify, a classification of different flow regimes in porous media is proposed by Dybbs and Edwards (1984) based on an experimental study of flow through beds of rods, spheres and complex rod bundles. They observed that the flow in porous media becomes fully turbulent, for a Re based on averaged pore velocity and averaged pore size greater than 300 . In addition to experimental studies, direct numerical simulations (DNS) of turbulent flow in porous media have also been used to investigate the pore-scale turbulence (Jin et al. 2015; Uth et al. 2016; Jin and Kuznetsov 2017). However, detailed experimental measurement and DNS are laborious due to the often complex structure of the pores resulting in time-consuming experimental procedures and high computational costs, respectively. These facts have motivated researchers to develop macroscopic turbulence models for high Re flow in porous media. Vafai et al. (2006) presented a thorough classification of turbulence models and divided these models into four categories based on the method of driving the governing equations for turbulent flow within porous media: (1) time averaging the transport equations in porous media (Antohe and Lage 1997; Getachew et al. 2000). It has been argued that this approach neglects the effect of turbulence within the pores (Nakayama and Kuwahara 1999), (2) volume averaging the Reynolds-averaged equations (Nakayama and Kuwahara 1999, 2008), (3) models based on morphology of porous media (Travkin and Catton 1992), and (4) models based on double decomposition concept 
(Pedras and de Lemos 2001). Some of these macroscopic models contain extra production terms that are modeled to account for the effect of the solid matrix on the turbulent flow. In addition to the models mentioned above, different versions of the macroscopic turbulence models have been reported in the literature such as the zero equation model (Masuoka and Takatsu 1996), the one equation model (Alvarez et al. 2003), the multi-scale four equations eddy viscosity model of Kuwata et al. (2014) and a model based on a second moment closure derived in Kuwata and Suga (2013).

Interesting enough, while there is a relatively large focus on the effects of pore-scale turbulence in porous media, there exists a hydrodynamic dispersion term which is usually neglected. Using the double decomposition concept, Pedras and de Lemos (2001) showed that the dispersion term materializes along with the averaged Reynolds stress after the averaging processes. Based on the study of Vafai and Tien (1981), Nakayama and Kuwahara $(1999,2008)$ claimed that the effect of hydrodynamic dispersion is included in the Darcy-Forchheimer term being used to model the viscous and form drags exerted on the fluid passing through the solid matrix. Also, Nield (1991) reported that the omission of the dispersion term is a reasonable assumption by showing that the inertia term in the momentum equation for porous media can be neglected as compared to the Forchheimer term containing the square of velocity. Despite the wide variety of the macroscopic models presented for pore-scale Reynolds stresses in porous media and assumptions that have been made to model the hydrodynamic dispersion, to the author's knowledge, none of the works reviewed above neither validated their assumptions nor compared their results with the values of these quantities averaged over the pores. This is maybe due to the complexity of experimental measurement of these quantities and DNS simulation of turbulent flow within the pores even for an ordered porous media which makes such a data scarce in the literature. Recently, Jin and Kuznetsov (2017) carried out a DNS study for turbulent flow within an array of spheres in the range of Re based on the sphere diameter between $260<\mathrm{Re}_{\mathrm{D}}<470$ and for the porosity between 0.69 and 0.8 . They showed that not only the dispersion term but also the shear components of Reynolds stress can be neglected due to their small values compared to the Forchheimer term. However, their conclusion is limited to the mentioned porosity and particle Re and there is still a need to study the pore-scale Reynolds stress and hydrodynamic dispersion in higher Re, a wider range of porosities and different geometries. Such a study gives a better understanding of the macroscopic effects of these phenomena on the momentum distribution in porous media and can be used for the validation of assumptions made to model or neglect these quantities.

Motivated by this, the present study aims to investigate the importance of the intra-pore Reynolds stress and hydrodynamic dispersion terms in the averaged momentum equation that are obtained from double decomposition. To fulfill this aim, a detailed numerical pore-scale study of turbulent flow in porous media that consists of spherical particles will be carried out. Four ordered unit cells and one non-ordered unit cell with different porosities are considered for the large eddy simulations performed. At first, vortical structures within the geometries of interest are visualized for $\operatorname{Re}_{H}=\rho U_{\mathrm{D}} H / \mu=1500$ and 15,000 to investigate the turbulence structures within the pores. Subsequently, the normal and shear components of the hydrodynamic dispersion and the Reynolds stress are evaluated, averaged and then compared for the two Re values. The averaged value of the hydrodynamic dispersion is also derived for a laminar flow case, $\operatorname{Re}_{H}=150$, to compare its value against the quadratic drag term. Then distributions of shear components of hydrodynamic dispersion and Reynolds stress within the pores are studied. Finally, a discussion is provided on the validity of eddy viscosity hypothesis and the effect of variation in pore-scale geometry on the turbulence structures and averaged values of dispersion and Reynolds stress. 


\section{Physical Model}

The simulations in the present study are carried out for a porous media formed by a 3D array of spheres, as shown in Fig. 1. A uniform macroscopic flow field is considered in the x-direction. The unit cell shown in Fig. 1a is treated as one cubic unit cell of a porous medium with the dimensions $H \times H \times H$ where nine spherical particles with the diameter $d$ are positioned at the corners and in the center of the cube. In order to study the effect of non-ordered placed particles on the hydrodynamic dispersion and Reynolds stress, a non-ordered geometry is also considered where an additional 10th sphere is placed at $(-0.266 \mathrm{H} 0.266 \mathrm{H}-0.166 \mathrm{H})$ with respect to the center of cube, as shown in Fig. 1b. Unit cells with different particle shapes have been extensively used in the literature to study the fluid flow through porous media such as LES and DNS study of turbulent flow in porous media (Kuwahara et al. 2006; Jin et al. 2015; Uth et al. 2016; Jin and Kuznetsov 2017; Chu et al. 2018), to calculate macroscopic quantities (Kundu et al. 2014; Yang et al. 2014) or to validate the macroscopic turbulence model in porous media (Nakayama and Kuwahara 1999; Pedras and de Lemos 2001). The simulations are carried out for five porosities, $\varphi=0.4,0.6,0.8,0.95$ and 0.94 (random) and for $\operatorname{Re}_{H} \simeq 150,1500$ and 15,000, which are defined as

$$
\begin{gathered}
\varphi=1-\frac{\pi d^{3}}{3 H^{3}}, \\
\operatorname{Re}_{\mathrm{D}}=\frac{\rho U_{\mathrm{D}} d}{\mu}, \operatorname{Re}_{H}=\frac{\rho U_{\mathrm{D}} H}{\mu},
\end{gathered}
$$

where $U_{\mathrm{D}}$ is the Darcian velocity which is defined as $U_{\mathrm{D}}=\varphi\langle U\rangle$.

\section{Governing Equations}

\subsection{Filtered Equations}

The filtered equations governing the problem under consideration are obtained by applying a filtering operation to the continuity and momentum equations, as follows:
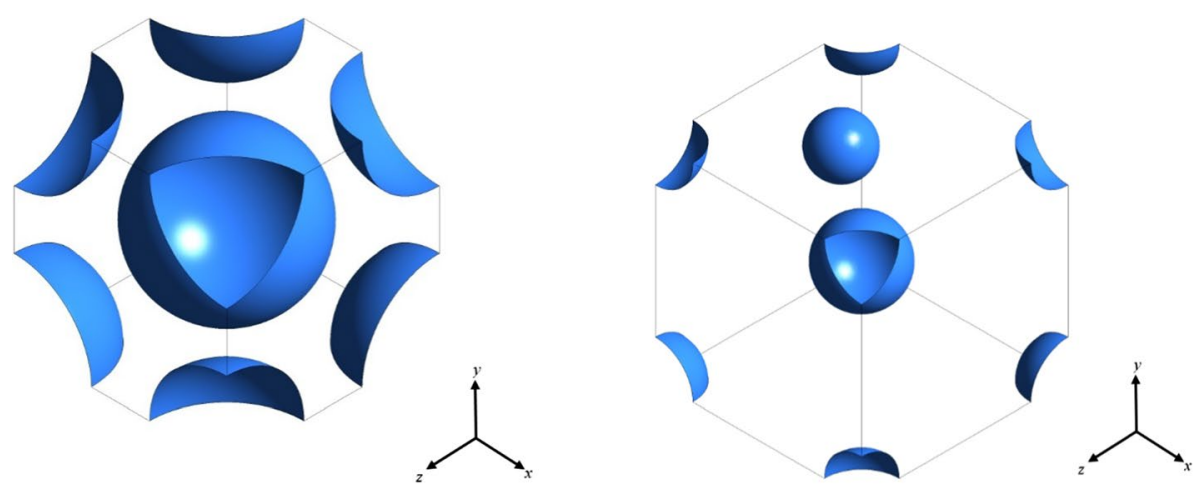

Fig. 1 The ordered and non-ordered unit cells in the present study 


$$
\begin{gathered}
\frac{\partial \bar{U}_{j}}{\partial x_{j}}=0 \\
\frac{\partial \bar{U}_{j}}{\partial t}+\frac{\partial \bar{U}_{i} \bar{U}_{j}}{\partial x_{i}}=-\frac{1}{\rho} \frac{\partial \bar{p}}{\partial x_{j}}+v \frac{\partial^{2} \bar{U}_{j}}{\partial x_{i} \partial x_{i}}-\frac{\partial \tau_{i j}^{r}}{\partial x_{i}}
\end{gathered}
$$

where $\tau_{i j}^{r}$ is the subgrid-scale (SGS) stress tensor, which includes the effects from the smallscale turbulence and is defined as

$$
\tau_{i j}^{r}=\overline{U_{i} U_{j}}-\bar{U}_{i} \bar{U}_{j}
$$

The SGS stress tensor is correlated with the filtered strain rate tensor using the eddy viscosity hypothesis as

$$
\tau_{i j}^{r}=-2 v_{\mathrm{sgs}} \bar{S}_{i j}
$$

where $\bar{S}_{i j}=\frac{1}{2}\left(\frac{\partial \overline{U_{i}}}{\partial x_{j}}+\frac{\partial \overline{U_{j}}}{\partial x_{i}}\right)$ and $v_{\mathrm{SGS}}$ are the filtered rate of the strain tensor and the eddy viscosity of the residual motions, respectively. The wall-adapted local eddy viscosity, WALE, model is used to model the SGS stress in this study as

$$
v_{\mathrm{sgs}}=\left(C_{\mathrm{w}} \Delta\right)^{2}\left(\frac{\left(S_{i j}^{d} S_{i j}^{d}\right)^{3 / 2}}{\left(\bar{S}_{i j} \bar{S}_{i j}\right)^{5 / 2}+\left(S_{i j}^{d} S_{i j}^{d}\right)^{5 / 4}}\right),
$$

where

$$
S_{i j}^{d}=\frac{1}{2}\left(\bar{g}_{i j}^{2}+\bar{g}_{j i}^{2}\right)-\frac{1}{3} \delta_{i j} \bar{g}_{k k}^{2}
$$

where $\bar{g}_{i j}=\partial \bar{U}_{i} / \partial x_{j}$. The value of $C_{\mathrm{w}}$ is set to 0.5 (Pope 2001; Ansys 2012). $\Delta$ is the filter cutoff width which defines the limit for the size of eddies that are resolved and those that are modeled. It is usually selected to be of the same order of the grid size (Versteeg and Malalasekera 2007). Assuming the grid cells with a different length $\Delta_{x}$, width $\Delta_{y}$ and height $\Delta_{z}$, the cutoff width is calculated as the cubic root of the cell volume as

$$
\Delta=\sqrt[3]{\Delta_{x} \Delta_{y} \Delta_{z}}
$$

\subsection{Volume-Averaged Equations for Flows in Porous Media}

Based on the double decomposition approach proposed by Pedras and de Lemos (2001), the space-time-averaged mass and momentum equations in porous media are expressed as

$$
\frac{\partial \varphi\left\langle\bar{U}_{j}\right\rangle}{\partial x_{j}}=0,
$$




$$
\frac{\partial\left\langle\bar{U}_{j}\right\rangle}{\partial t}+\frac{1}{\varphi} \frac{\partial}{\partial x_{i}}\left(\varphi \overline{\left\langle U_{i} U_{j}\right\rangle}\right)=-\frac{1}{\varphi \rho} \frac{\partial}{\partial x_{j}}(\varphi\langle\bar{p}\rangle)+\frac{v}{\varphi} \frac{\partial^{2}\left(\varphi\left\langle\bar{U}_{j}\right\rangle\right)}{\partial x_{i} \partial x_{i}}+\frac{\tilde{v}}{\varphi} \frac{\partial\left(\varphi\left\langle\bar{U}_{j}\right\rangle\right)}{\partial x_{i}}+f_{j}
$$

where $f_{j}$ takes into account the viscous and form drags excreted on the fluid passing through the porous matrix which is modeled by the Darcy-Forchheimer (Dupuit) expression as

$$
f_{j}=-\frac{v}{K} \varphi\left\langle\bar{U}_{j}\right\rangle-b \varphi^{2}\left(\left\langle\bar{U}_{i}\right\rangle\left\langle\bar{U}_{i}\right\rangle\right)^{1 / 2}\left\langle\bar{U}_{j}\right\rangle .
$$

The $\tilde{v}$ in Eq. (10) is the effective viscosity in the Brinkman modification. In the above equations, $\bar{\phi}, \phi^{\prime},\langle\phi\rangle$ and $\tilde{\phi}$ denote the time averages, time fluctuations, volume averaged and fluctuations from volume-averaged value of a general parameter $\phi$. The double decomposition of a general parameter $\phi$ may, therefore, be written as

$$
\phi=\langle\bar{\phi}\rangle+\left\langle\phi^{\prime}\right\rangle+\tilde{\bar{\phi}}+\bar{\phi}^{\prime},
$$

with the usage of the following relationships:

$$
\overline{\langle\phi\rangle}=\langle\bar{\phi}\rangle,\left\langle\phi^{\prime}\right\rangle=\langle\phi\rangle^{\prime}, \tilde{\bar{\phi}}=\overline{\tilde{\phi}}, \tilde{\phi}^{\prime}=\tilde{\phi}^{\prime} .
$$

According to Pedras and de Lemos (2001), the order of time and volume averaging is irrelevant for the momentum equation. Following Pedras and de Lemos (2001) and starting with time decomposition yields

$$
\begin{aligned}
\frac{\partial}{\partial x_{i}}\left(\varphi \overline{\left\langle U_{i} U_{j}\right\rangle}\right) & =\frac{\partial}{\partial x_{i}}\left(\overline{\varphi\left\langle\left(\bar{U}_{i}+U_{i}^{\prime}\right)\left(\bar{U}_{j}+U_{j}^{\prime}\right)\right\rangle}\right) \\
& =\frac{\partial}{\partial x_{i}}\left(\varphi\left(\left\langle\bar{U}_{i} \bar{U}_{j}\right\rangle+\left\langle\overline{U_{i}^{\prime} U_{j}^{\prime}}\right\rangle\right)\right)=\frac{\partial}{\partial x_{i}}\left(\varphi\left(\left\langle\bar{U}_{i}\right\rangle\left\langle\bar{U}_{j}\right\rangle+\left\langle\tilde{U}_{i} \tilde{U}_{j}\right\rangle+\left\langle\overline{U_{i}^{\prime} U_{j}^{\prime}}\right\rangle\right)\right) \\
& =\frac{\partial}{\partial x_{i}}\left(\varphi\left(\left\langle\bar{U}_{i}\right\rangle\left\langle\bar{U}_{j}\right\rangle+\left\langle\tilde{\tilde{U}}_{i} \tilde{\tilde{U}}_{j}\right\rangle+\left\langle\overline{\left\langle U_{i}^{\prime}\right\rangle\left\langle U_{j}^{\prime}\right\rangle}+\left\langle\overline{\tilde{U}_{i}^{\prime} \tilde{U}_{j}^{\prime}}\right\rangle\right)\right) .\right.
\end{aligned}
$$

By instead starting from volume decomposition, the following expression is derived:

$$
\begin{aligned}
\frac{\partial}{\partial x_{i}}\left(\varphi \overline{\left\langle U_{i} U_{j}\right\rangle}\right) & =\frac{\partial}{\partial x_{i}}\left(\overline{\varphi\left\langle\left(\left\langle U_{i}\right\rangle+\tilde{U}_{i}\right)\left(\left\langle U_{j}\right\rangle+\tilde{U}_{j}\right)\right\rangle}\right) \\
& =\frac{\partial}{\partial x_{i}}\left(\overline{\varphi\left(\left\langle U_{i}\right\rangle\left\langle U_{j}\right\rangle+\left\langle\tilde{U}_{i} \tilde{U}_{j}\right\rangle\right)}\right) \\
& =\frac{\partial}{\partial x_{i}}\left(\overline{\varphi\left[\left(\left\langle\bar{U}_{i}\right\rangle+\left\langle U_{i}^{\prime}\right\rangle\right)\left(\left\langle\bar{U}_{j}\right\rangle+\left\langle U_{j}^{\prime}\right\rangle\right)+\left\langle\tilde{U}_{i} \tilde{U}_{j}\right\rangle\right]}\right) \\
& =\frac{\partial}{\partial x_{i}}\left(\varphi\left(\left\langle\bar{U}_{i}\right\rangle\left\langle\bar{U}_{j}\right\rangle+\overline{\left\langle U_{i}^{\prime}\right\rangle\left\langle U_{j}^{\prime}\right\rangle}+\overline{\left\langle\tilde{U}_{i} \tilde{U}_{j}\right\rangle}\right)\right) \\
& =\frac{\partial}{\partial x_{i}}\left(\varphi(\underbrace{\left\langle\bar{U}_{i}\right\rangle\left\langle\bar{U}_{j}\right\rangle}_{I}+\underbrace{\overline{\left\langle U_{i}^{\prime}\right\rangle\left\langle U_{j}^{\prime}\right\rangle}}_{I I}+\underbrace{\left\langle\tilde{U}_{i} \tilde{\tilde{U}}_{j}\right\rangle}_{I I I}+\underbrace{\left\langle\overline{\tilde{U}_{i}^{\prime} \tilde{U}_{j}^{\prime}}\right\rangle}_{I V})\right) .
\end{aligned}
$$


As can be observed from Eqs. (15) and (16), the final form of the two approaches are identical. It is, in this context, worth to mention that the Reynolds stress in porous media, which is usually discussed in the literature, is the sum of terms $I I$ and $I V$ as

$$
\rho \varphi\left\langle\overline{U_{i}^{\prime} U_{j}^{\prime}}\right\rangle=\underbrace{\overline{\left\langle U_{i}^{\prime}\right\rangle\left\langle U_{j}^{\prime}\right\rangle}}_{I I}+\underbrace{\left\langle\overline{\tilde{U}_{i}^{\prime} \tilde{U}_{j}^{\prime}}\right\rangle}_{I V},
$$

which is commonly modeled as

$$
\rho \varphi\left\langle\overline{U_{i}^{\prime} U_{j}^{\prime}}\right\rangle=\mu_{t \varphi}\left[\frac{\partial\left(\varepsilon\langle\bar{U}\rangle_{i}\right)}{\partial x_{j}}+\frac{\partial\left(\varepsilon\langle\bar{U}\rangle_{j}\right)}{\partial x_{i}}\right]-\frac{2}{3} \rho \varphi\langle k\rangle \delta_{i j} .
$$

According to Pedras and de Lemos (2001), the physical significance of the four terms on the RHS of Eq. (16) is as follows:

(I) Convection of macroscopic mean velocity.

(II) Macroscopic Reynolds stress due to the fluctuation of macroscopic velocity.

(III) Dispersion due to space fluctuation of time mean velocity within the pores present in both laminar and turbulent flows.

(IV) Dispersion due to both time and space fluctuation of velocity within the pores.

\section{Numerical Simulation and Boundary Condition}

The governing equations are solved using the commercial code Ansys CFX 16.2. The parallelization of the computational domain is carried out using the MeTis partitioning method and simulations are carried out on a LINUX cluster applying MPI-4.1.3 messagepassing libraries (MPI) (Hellström et al. 2007). The bounded central difference is used to discretize the advection terms while the unsteady terms are discretized using the secondorder backward Euler scheme. The time step is selected so that the Courant number falls between 0 and 1 . Symmetry boundary conditions are applied on the bottom, top and side boundaries, periodic boundary conditions are set at the inlet and outlet and no-slip boundary conditions are used on the walls of the spheres.

\section{Grid Independency, Verification and Validation}

The grid independence study is done using three element sizes for each porosity as shown in Fig. 2. It can be observed that the time-averaged velocity distribution along the y-direction is very close to each other in all studies which are exemplified in Fig. 2 for $\varphi=0.95$ and $\varphi=0.6$. The largest number of elements is still chosen for the rest of the simulations in this study since $y^{+}$below 1 is guaranteed for this case. As a result, element meshes of approximately $1.8 \times 10^{6}, 1.9 \times 10^{6}, 1.7 \times 10^{6}$ and $1.2 \times 10^{6}$ are constructed for $\varphi=0.95$, $0.8,0.6$ and 0.4 , respectively.

As a sort of validation of the simulations, the results of volume-averaged turbulent kinetic energy are compared with those obtained with LES in Kuwahara et al. (2006) for 


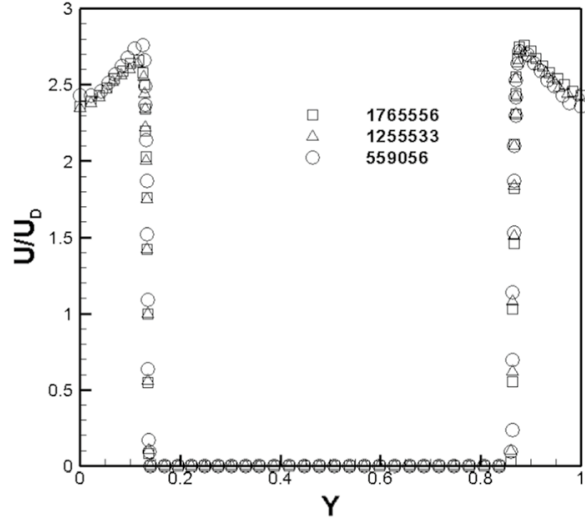

(a)

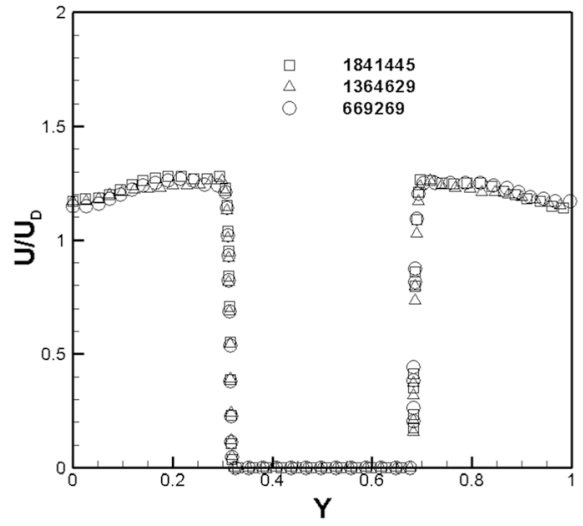

(b)

Fig. 2 Two examples of grid independence study in $\mathbf{a} \varphi=0.6, \mathbf{b} \varphi=0.95$

turbulent flow in an array of staggered square cylinders and the $k-\varepsilon$ modeling of turbulent flow as reported in Yang et al. (2014) for the array in Fig. 1. As can be observed, the values obtained in the present study are in a good agreement with those reported by Yang et al. (2014) and lower than that obtained in the LES study of Kuwahara et al. (2006) which is due to square geometry of their cylinders cross section which enhances the production of turbulent kinetic energy. This is also observed by Yang et al. (2014) where the values obtained by $k-\varepsilon$ modeling of turbulent flow in an array similar to that in Fig. 1 are significantly lower than the results from the $k-\varepsilon$ simulation reported by Nakayama and Kuwahara (1999) for the staggered arrangement of square cylinders, see Table 1.

Further validation of the present results is carried out in Table 2 where the Blake-type friction factor, as derived in the present study, is compared with that of Ergun and Orning (1949) and numerical simulation of turbulent flow in an array of cylinders reported in Hellström et al. (2010). The Blake-type friction factor is calculated as

$$
f^{\prime}=\frac{\Delta p}{L} \frac{d}{\rho \varphi^{2}\langle U\rangle^{2}} \frac{\varphi^{3}}{1-\varphi} .
$$

Introducing this correlation into the Ergun experimental relationship yields

Table 1 Comparison of the averaged turbulent kinetic energy

\begin{tabular}{lllll}
\hline$\langle k\rangle$ & LES present & $\begin{array}{l}k-\varepsilon \text { (Yang } \\
\text { et al. 2014) }\end{array}$ & $k-\varepsilon(\mathrm{N}-\mathrm{K})$ & $\begin{array}{l}\text { LES (Kuwa- } \\
\text { hara et al. } \\
\text { 2006) }\end{array}$ \\
Geometry & Figure 1 & & $\begin{array}{l}\text { Staggered arrangement of } \\
\text { square cylinders }\end{array}$ \\
\hline$\varphi=0.4$ & 1.34 & $0.54^{\mathrm{a}}$ & 3.5 & 2.1 \\
$\varphi=0.6$ & 0.36 & 0.297 & 1.91 & 1.2 \\
$\varphi=0.8$ & 0.145 & 0.14 & 0.82 & 0.5 \\
$\varphi=0.95$ & 0.039 & 0.039 & 0.18 & Not reported \\
\hline
\end{tabular}

${ }^{\text {a }}$ This value is not in the range of the simulations performed by Yang et al. (2014) and is calculated using the fitted correlation proposed in their study 
Table 2 Comparison of the Blake-type friction factor

\begin{tabular}{lrlll}
\hline Case & $\mathrm{Re}^{\prime}$ & $f^{\prime}$ Present & $f^{\prime}$ (Hellström et al. 2010) & $f^{\prime}$ Ergun \\
\hline$\varphi=0.4$ & 213 & 1.35 & 1.65 & 2.45 \\
& 2131 & 1.02 & 0.89 & 1.82 \\
& 21,317 & 0.42 & 0.47 & 1.75 \\
$\varphi=0.6$ & 279 & 1.08 & 0.87 & 2.25 \\
& 2795 & 0.67 & $0.39\left(\operatorname{Re}^{\prime}=1000\right)$ & 1.8 \\
& 27,950 & 0.28 & Not reported & 1.75 \\
\hline \multicolumn{5}{c}{$=1.75+\frac{150}{\operatorname{Re}^{\prime}}, \operatorname{Re}^{\prime}=\frac{\rho \varphi\langle U\rangle d}{\mu(1-\varphi)}}$.
\end{tabular}

Since the porosity of packed beds usually is low, the comparison is only shown for $\varphi=0.4$ and $\varphi=0.6$. As can be observed, the present results are closer to the numerical simulation in Hellstöm et al. (2010) at higher Re while both numerical simulations predict values lower than those obtained by the correlation based on experimental measurements of Ergun and Orning (1949). Hellstöm et al. (2010) discussed that the turbulence effects must be included in the simulations for $\mathrm{Re}^{\prime}$ larger than of the order of 100 . Neglecting turbulence results in an underprediction of the friction factor.

\section{Results and Discussion}

At first, the flow field is studied in different geometries and Re. Then the importance of the hydrodynamic dispersion and the Reynolds stress is discussed by averaging their values over the unit cells as shown in Fig. 1. In several of the plots presented, the $Q$-criterion is used to reveal vortical structures in the flow. The value of $Q$ is defined as the second invariant of the velocity gradient tensor and its positive values identify vortical structures. Notice that the values of $Q$ that discloses the vortical structures vary between the porosities and Re. Therefore, the $Q$ values presented in this work are normalized with the maximum value of $Q, Q_{M}$, in each case, following Uth et al. (2016). The iso-surfaces of $Q$ are also colored with the $y$-velocity to show their motion.

\subsection{Pore-Scale Flow Field}

The vortical structures become more fragmented as $\mathrm{Re}_{\mathrm{H}}$ is increased from 1500 to 15,000 for all porosities studied, see Fig. 3. In the case of $\varphi=0.95$ and $\varphi=0.94$, these structures are nearly similar to those observed for turbulent flow passing over one sphere (Rodriguez et al. 2011; Rodríguez et al. 2018). This is due to the larger space between the particles for this porosity. Also, a similar tendency to form a hairpin vortex as reported in Prahl et al. (Prahl et al. 2009) for an in-line arrangement of spheres can be seen as the vortex formed by the upstream sphere passes around the trailing sphere for the larger porosities and $\operatorname{Re}_{H}=1500$. Examples of such structures are indicated by the black arrows in Fig. 3 for $\operatorname{Re}_{H}=1500$ and $\varphi=0.95, \varphi=0.94$ and $\varphi=0.8$. It can be observed that large vortices break into smaller ones and form a turbulent wake behind the spheres as the Re increases to $\operatorname{Re}_{H}=15000$ for larger porosities, as shown with the black open arrows in Fig. 3. As can be seen in Fig. 3, the length scale of the vortical structures is of the same order of or smaller than the pore size in all porosities and Re studied here. Although some of them 


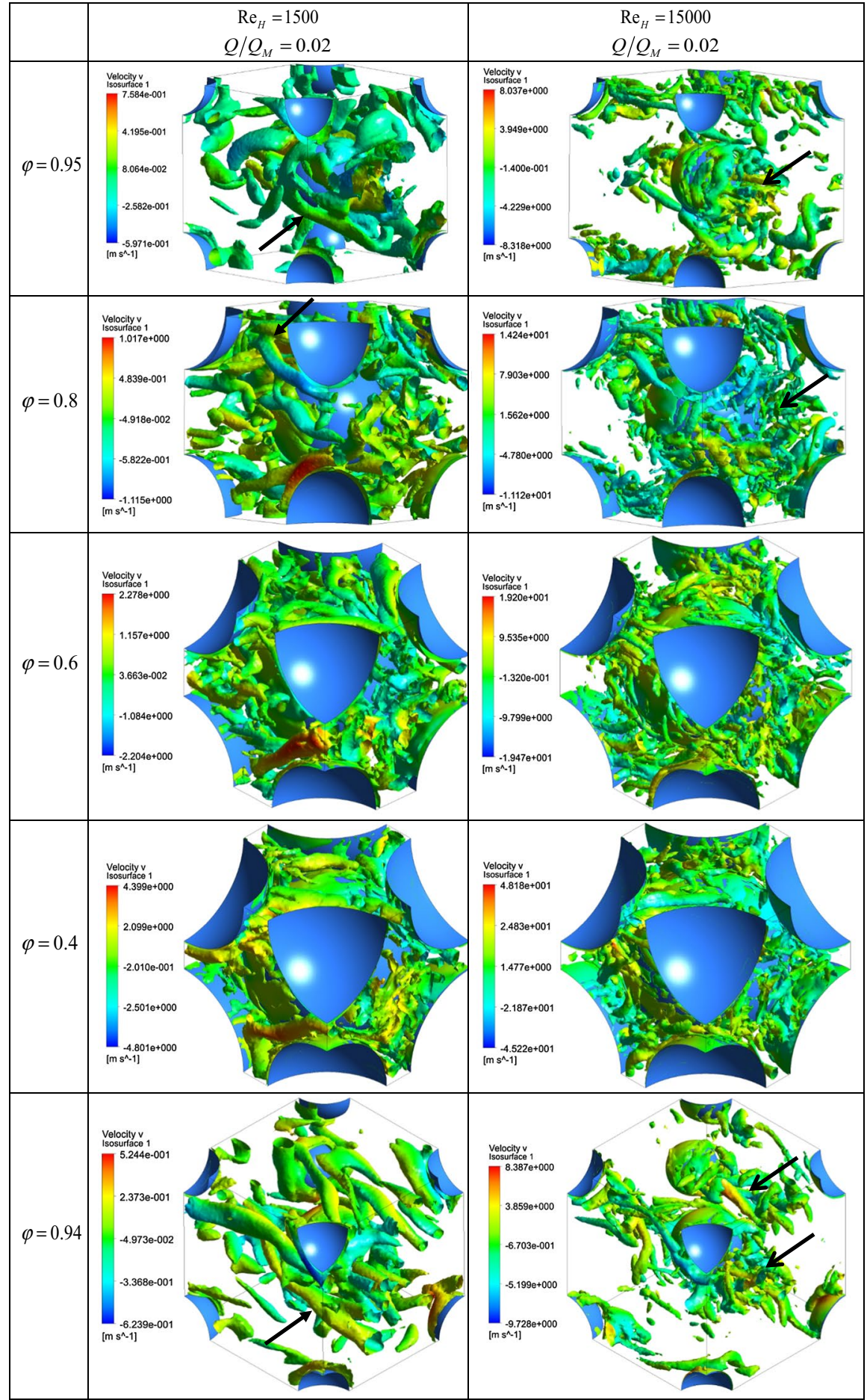

Fig. 3 Vortical structures within the unit cell identified by iso-surfaces of $Q$ for a different $\varphi$ and $\operatorname{Re}$ 
penetrate into the neighboring pores, as can be seen from the periodicity of some vortical structures, still their size is smaller than the pore dimension. In order to investigate the effect of randomness of the solid structure on turbulence structures in the pores, one sphere is added to the unit cell with $\varphi=0.95$ which reduces slightly its porosity to $\varphi=0.94$. A trend similar to that observed for $\varphi=0.95$ can be observed for $\varphi=0.94$ and the added particle does not change the general form of the turbulence structures within the pores, see Fig. 3. The effect of the number of unit cells on the size of the turbulent structures is studied by considering turbulent flow in two unit cells placed in parallel and in series with respect to the main flow direction for the case of $\varphi=0.6$ and $\operatorname{Re}_{H}=1500$. As can be observed in Fig. 4, adding these unit cells do not affect the shape of the turbulent structures and these structures are similar to those obtained in Fig. 3 for one unit cell. The present results show that pore-scale prevalence hypothesis of Uth et al. (2016) is still valid for the range of porosities between 0.4 and $0.95, \mathrm{Re}_{H}=15000$ and also for a random geometry.

\subsection{Averaged Hydrodynamic Dispersion and Reynolds Stress}

The LES study of turbulent flow within the pores of porous media provides the opportunity to directly evaluate the volume-averaged values of hydrodynamic dispersion and Reynolds stress, term III and term II + IV [from Eq. (17)], on the RHS of Eq. (15), respectively. As can be seen in Figs. 5 and 6, the normal and shear components of the volume-averaged hydrodynamic dispersion are of the same order as their Reynolds stress counterparts for all porosities and Re investigated. In order to investigate the effects of dispersion on the averaged momentum equations in laminar flow, the hydrodynamic dispersion is calculated for $\operatorname{Re}_{H}=150$ in Fig. 7. Notice that all of the values in these figures are normalized with the square of the Darcy velocity. As can be seen in Figs. 5b, $6 \mathrm{~b}$ and $7 \mathrm{~b}$, the shear components of both the hydrodynamic dispersion and the Reynolds stress are small in all Re and porosities studied here and vary non-monotonically with porosity and Re while the normal components are not negligible and decrease with an increase in porosity. It can be observed from Fig. $7 \mathrm{~b}$ that the longitudinal component of hydrodynamic dispersion $(u u)$ is relatively large in $\operatorname{Re}_{H}=150$. This can imply that the longitudinal dispersion can highly affect the flow through porous media (Sozen and Vafai 1993). Meanwhile, Amiri and Vafai (1994) reported that the transverse components of hydrodynamic dispersion ( $v v$ and $w w$ ) may play a more important role in heat transfer through porous media despite their lower values. Figure 8 illustrates the variation in hydrodynamic dispersion and Reynolds stress with Re. As can be seen in most cases, the normal components of the Reynolds stress increase slightly with an increase in Re from 1500 to 15,000 . It can be observed that the normal components of hydrodynamic dispersion in the longitudinal direction $(u u)$ dominate over other normal components and decrease with an increase in $\operatorname{Re}$ for $\varphi=0.4$ and $\varphi=0.6$. Also, in the cases of $\varphi=0.8$ and $\varphi=0.95$, a slight increase is observed with the increase in Re from 150 to 1500 followed by a decrease as the Re is increased to 15,000 . Such a trend is observed for the nonuniformity in the spatial distribution of time-averaged velocity in Khayamyan et al. (2017a). In their work, the initial increase with Re is attributed to the enhancement of inertial effects as the flow regime changes from creeping flow to transitional regime while the subsequent reduction with an increase in Re is assumed to be due to the turbulence effects becoming dominated which enhance mixing resulting in more uniform distribution. It is predicted that this phenomenon may be reflected in macroscopic quantities such as dispersion, as observed in Figs. 5, 6 and 7. The results obtained here shed some light on the shear components of the tensors in Eq. (15). The results presented in Figs. 5b, $6 \mathrm{~b}$ and $7 \mathrm{~b}$ suggest 


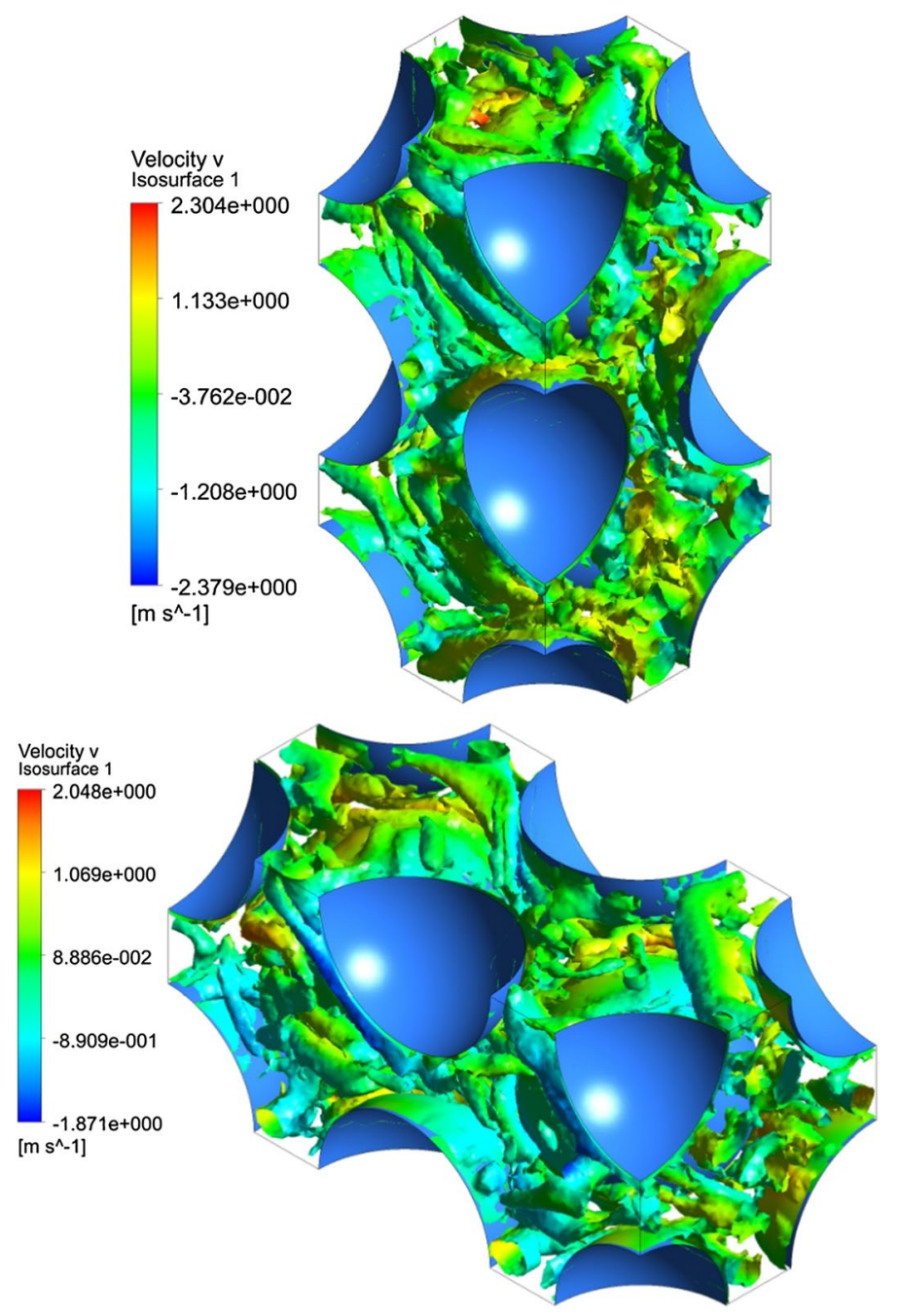

Fig. 4 Vortical structures within two unit cells placed in parallel and in series, respectively, with respect to the main flow direction for $\varphi=0.6$ and $\operatorname{Re}_{H}=1500$. The structures are identified by iso-surfaces of $Q$ $\left(Q / Q_{M}=0.02\right)$

that the shear components, containing two space fluctuating velocities, may be normally distributed in space with a mean value close to zero. This conclusion is based on the fact that the averaged values of the shear components in Figs. $5 \mathrm{~b}, 6 \mathrm{~b}$ and $7 \mathrm{~b}$ are negligible and have positive and negative values without any specific trend with regard to porosity or Re. The validity of this conclusion will be examined in the following section.

As mentioned above, the results presented in Figs. 5, 6 and 7 are normalized with $U_{\mathrm{D}}^{2}$. Since the Forchheimer term in the averaged momentum equation is a function of the $U_{\mathrm{D}}^{2}$, the negligible values of normalized dispersion and Reynolds stress shear components may suggest that these components are negligible compared to the Forchheimer term. The validity of this conclusion can be further examined by evaluating the ratio of the 


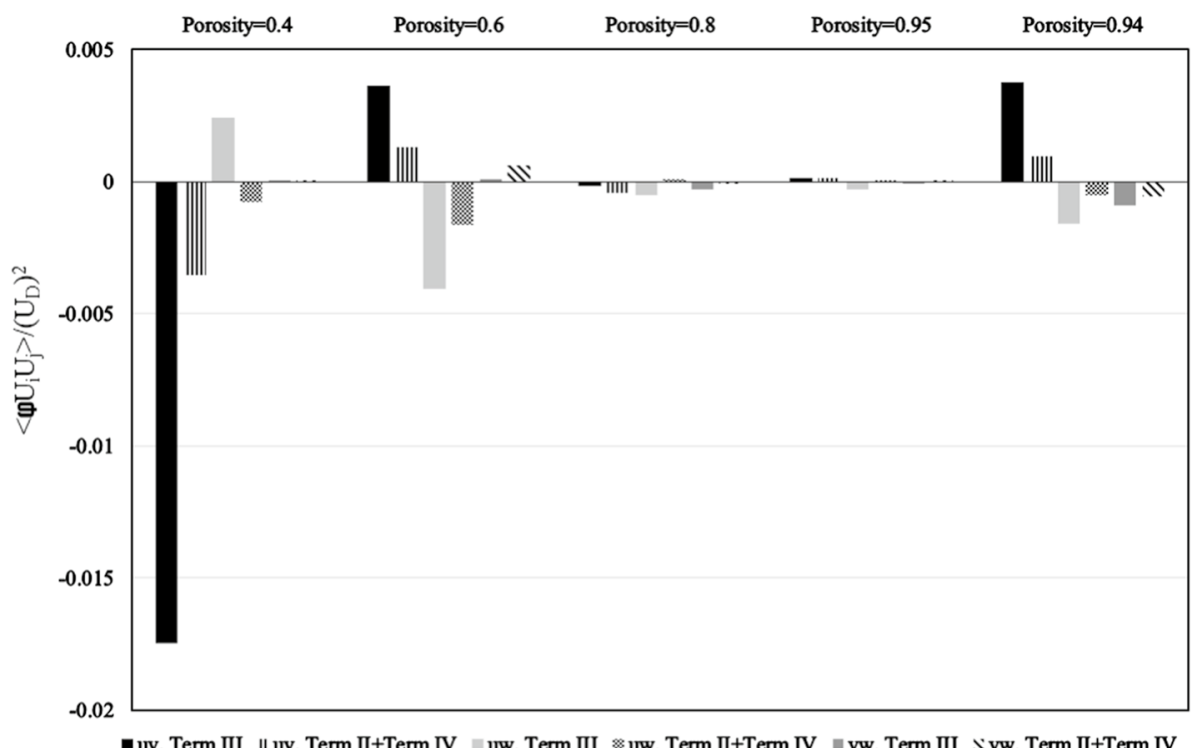

(a)

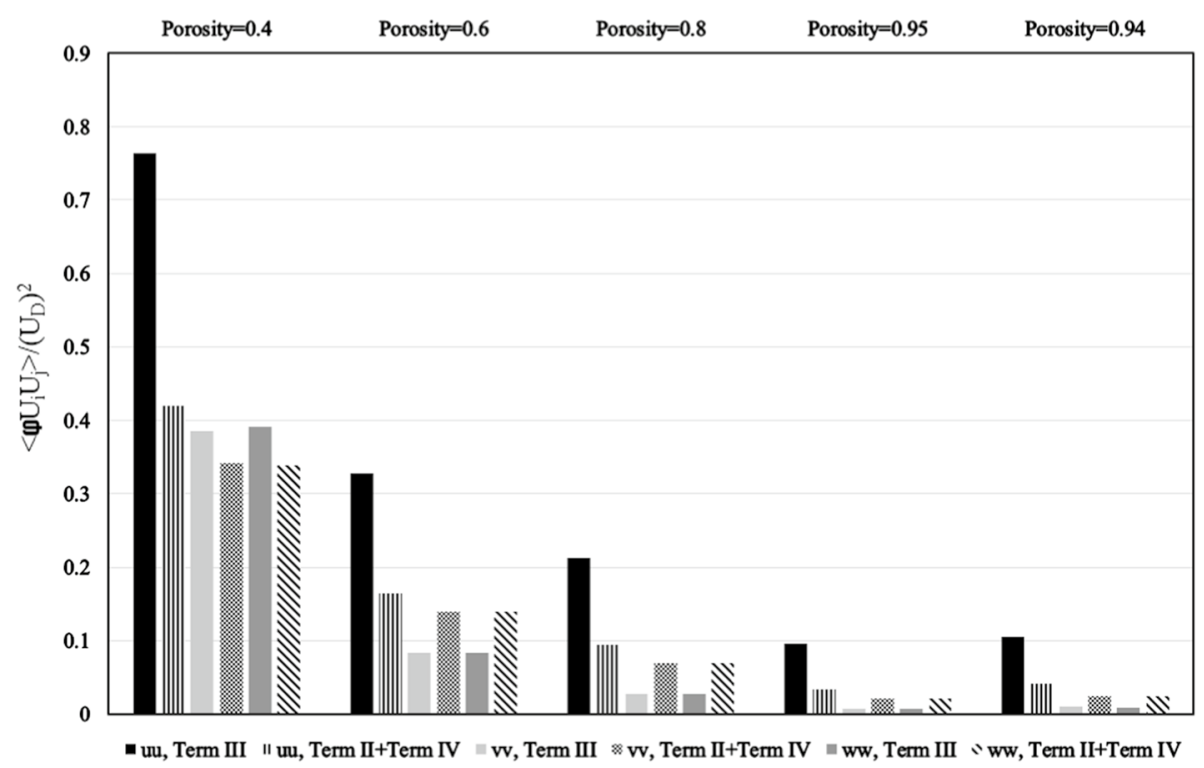

(b)

Fig. 5 Volume-averaged values of hydrodynamic dispersion and Reynolds stress at $\operatorname{Re}_{H}=15000$ a shear components, b normal components

gradients of volume-averaged Reynolds stress and dispersion shear components to the Forchheimer pressure drop. To this end, it is necessary to assume a condition in which the gradient of the volume-averaged Reynolds stress and dispersion terms are nonzero. Consider an extreme case where the volume-averaged macroscopic Reynolds stress and 


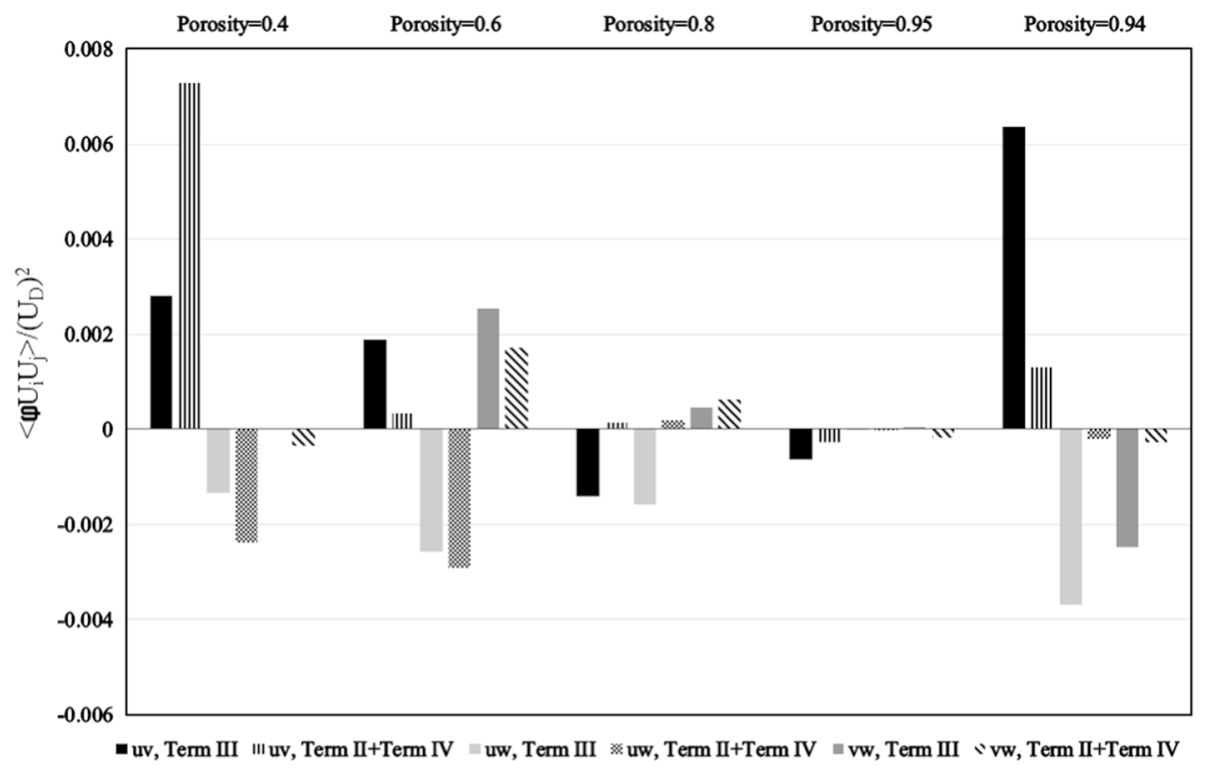

(a)

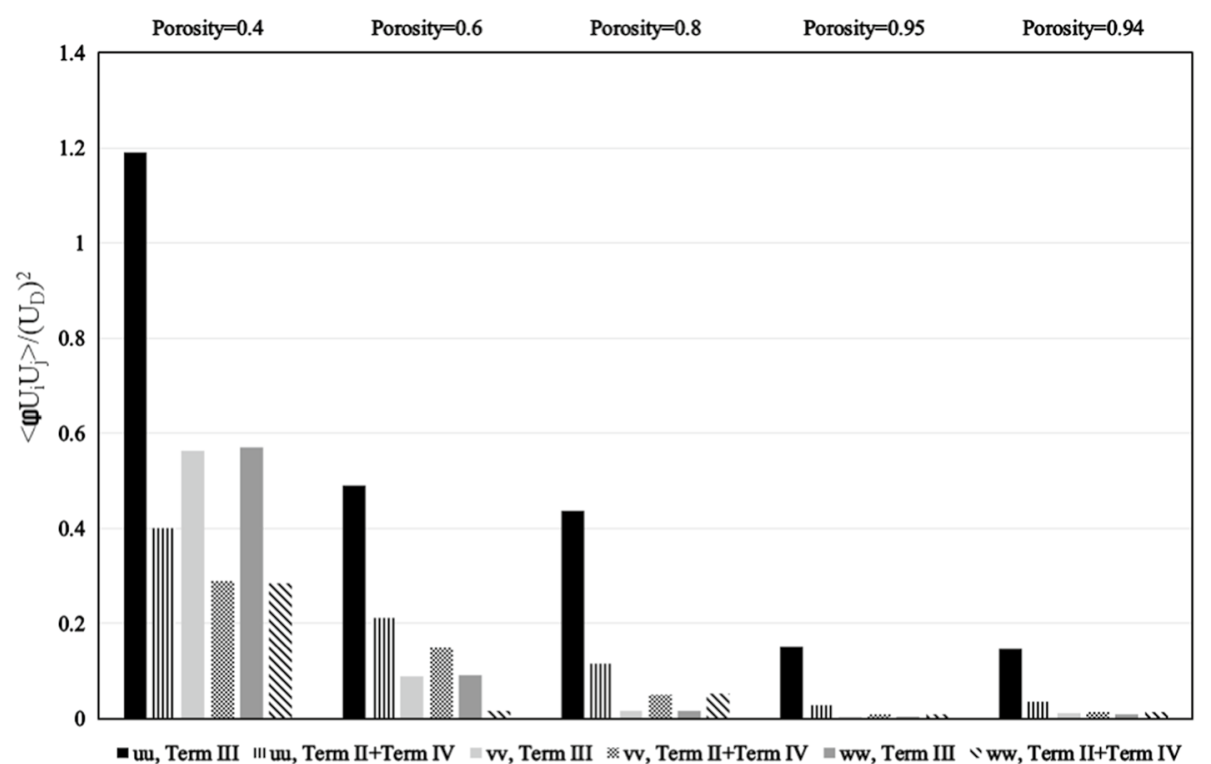

(b)

Fig. 6 Volume-averaged values of hydrodynamic dispersion and Reynolds stress at $\operatorname{Re}_{H}=1500$ a shear components, b normal components

dispersion reduce to zero in a neighboring unit cell which is $H$ away. For this case, the gradients of these quantities reach their maximum and the ratio of gradients of Term III and Term II + Term IV to the Forchheimer term will be given as 


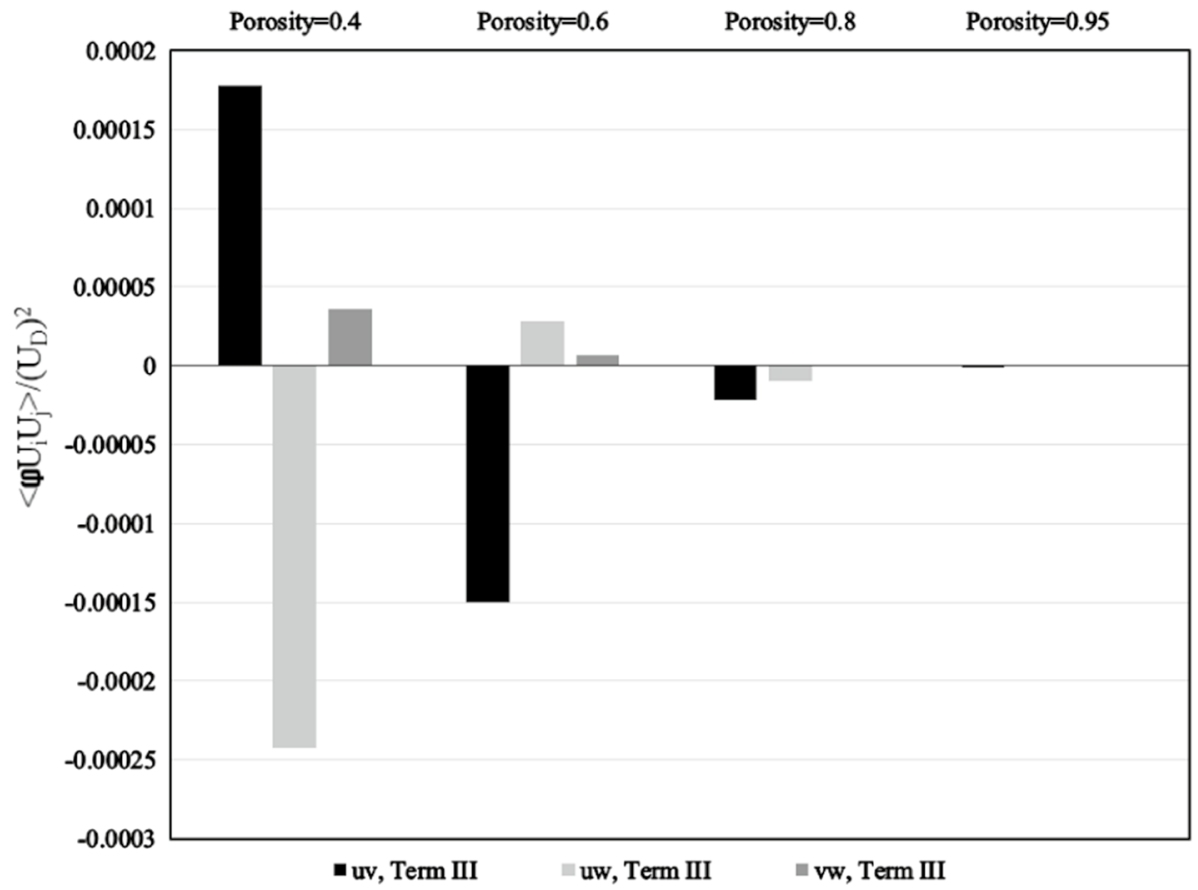

(a)

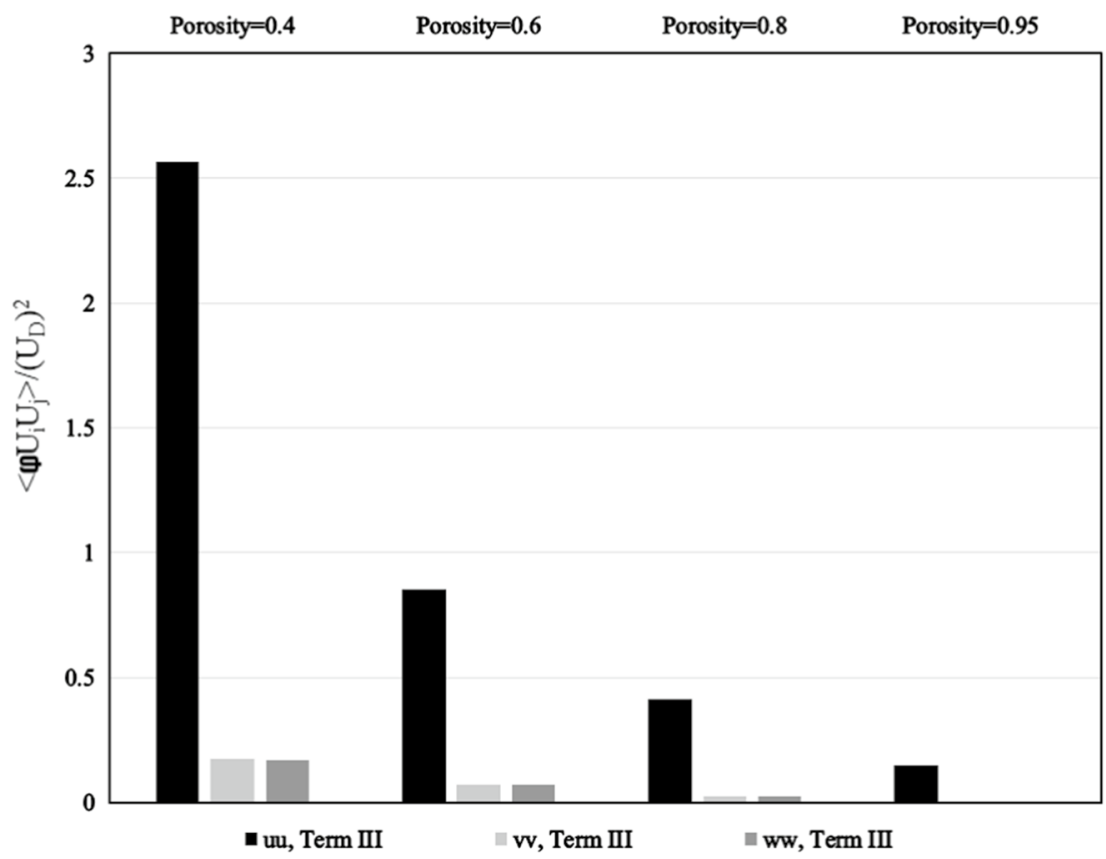

(b)

Fig. 7 Volume-averaged values of hydrodynamic dispersion at $\mathrm{Re}_{H}=150$ a shear components, $\mathbf{b}$ normal components 




Fig. 8 Variation in normal and shear components of hydrodynamic dispersion and Reynolds stress with Re

$$
\begin{gathered}
\frac{\partial\left(\overline{\left\langle U_{i}^{\prime}\right\rangle\left\langle U_{j}^{\prime}\right\rangle}+\left\langle\overline{\tilde{U}_{i}^{\prime} \tilde{U}_{j}^{\prime}}\right\rangle\right)}{\partial x_{i}} \approx \frac{\left(\overline{\left\langle U_{i}^{\prime}\right\rangle\left\langle U_{j}^{\prime}\right\rangle}+\left\langle\overline{\tilde{U}_{i}^{\prime} \tilde{U}_{j}^{\prime}}\right\rangle\right)}{H} \\
\frac{\partial(\text { Term } I I+\text { Term } I V) / \partial x_{i}}{\text { Forchheimer Term }}=\frac{\frac{\partial \varphi\left(\overline{\left\langle U_{i}^{\prime}\right\rangle\left\langle U_{j}^{\prime}\right\rangle}+\left\langle\overline{\tilde{U}_{i}^{\prime} \tilde{U}_{j}^{\prime}}\right\rangle\right)}{\partial x_{i}}}{b \varphi^{2}\left(\left\langle\bar{U}_{i}\right\rangle\left\langle\bar{U}_{i}\right\rangle\right)^{1 / 2}\left\langle\bar{U}_{j}\right\rangle} \approx \frac{\frac{\varphi\left(\overline{\left\langle U_{i}^{\prime}\right\rangle\left\langle U_{j}^{\prime}\right\rangle}+\left\langle\overline{\tilde{U}_{i}^{\prime} \tilde{U}_{j}^{\prime}}{ }_{j}\right\rangle\right)}{H}}{b \varphi^{2}\left(\left\langle\bar{U}_{i}\right\rangle\left\langle\bar{U}_{i}\right\rangle\right)^{1 / 2}\left\langle\bar{U}_{j}\right\rangle} \\
\approx \frac{1}{b H} \frac{\varphi\left(\overline{\left\langle U_{i}^{\prime}\right\rangle\left\langle U_{j}^{\prime}\right\rangle}+\left\langle\overline{\tilde{U}_{i}^{\prime} \tilde{U}_{j}^{\prime}}{ }_{j}\right\rangle\right)}{U_{D}^{2}}
\end{gathered}
$$




$$
\frac{\partial(\text { Term } I I I) / \partial x_{i}}{\text { Forchheimer Term }}=\frac{\frac{\partial \varphi\left\langle\tilde{U}_{i} \tilde{U}_{j}\right\rangle}{\partial x_{i}}}{b \varphi^{2}\left(\left\langle\bar{U}_{i}\right\rangle\left\langle\bar{U}_{i}\right\rangle\right)^{1 / 2}\left\langle\bar{U}_{j}\right\rangle} \approx \frac{\frac{\varphi\left\langle\tilde{U}_{i} \tilde{U}_{j}\right\rangle}{H}}{b \varphi^{2}\left(\left\langle\bar{U}_{i}\right\rangle\left\langle\bar{U}_{i}\right\rangle\right)^{1 / 2}\left\langle\bar{U}_{j}\right\rangle} \approx \frac{1}{b H} \frac{\varphi\left\langle\tilde{U}_{i} \tilde{U}_{j}\right\rangle}{U_{D}^{2}} .
$$

Based on the results presented in Figs. 5, 6 and 7 and $1 / b H$ values derived using $b=150(1-\varphi) / \varphi^{3} d$ and Eq. (1), the ratios of Eqs. (22) and (23) are presented in Fig. 9. Hence, the terms containing the gradients of the -veraged Reynolds stress and dispersion shear components are negligible compared to the Forchheimer term in the averaged momentum equation. It should be noted that only the Forchheimer term is considered in Eqs. (22) and (23) since viscous effects are negligible compared to the form drag (Forchheimer term) for the Re investigated according to Ergun correlation (1949) and LES results in Kuwahara et al. (2006). Jin and Kuznetsov (2017) showed that the shear components of intra-pore Reynolds stress do not affect the macroscopic transport of flow properties due to their negligible averaged values and their net local effect is to increase the averaged drag and therefore pressure drop. Hellström et al. (2010) numerically studied the effect of intra-pore turbulence and showed that ignoring the turbulence within the pores results in an underprediction in the pressure drop and friction factor.

\subsection{Discussion on the Distribution of Intra-pore Reynolds Stress and Hydrodynamic Dispersion}

In order to examine the distribution of the shear components of the tensors in Eq. (15) in this section, double decomposition of the $i=1, j=2$ component of $\left\langle U_{i} U_{j}\right\rangle$ is considered keeping in mind that the results will be similar for the case when $i=1, j=3$. A double decomposition of $\overline{\left\langle U_{1} U_{2}\right\rangle}$ yields

$$
\begin{aligned}
& \frac{\partial}{\partial x_{i}}\left(\varphi \overline{\left\langle U_{1} U_{2}\right\rangle}\right)=\frac{\partial}{\partial x_{i}}\left(\overline{\varphi\left(\left\langle U_{1}\right\rangle\left\langle\left\langle X_{2}\right\rangle+\left\langle\tilde{U}_{1} \tilde{U}_{2}\right\rangle\right)\right.}\right)
\end{aligned}
$$

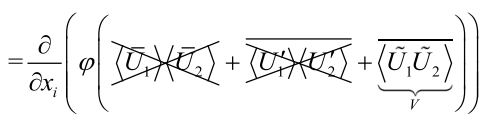

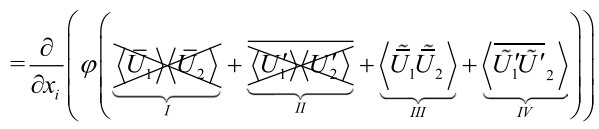

By volume averaging of the velocity components over the unit cells of Fig. 1, it is found that the only nonzero component of velocity is the one in the x-direction $\left(\left\langle\bar{U}_{1}\right\rangle \neq 0\right)$ while $\left\langle\bar{U}_{i}\right\rangle_{i=2, i=3} \approx 0$ and $\left\langle U_{i}^{\prime}\right\rangle \approx 0$. Therefore, the terms $I$ and $I I$ that correspond to convection and macroscopic Reynolds stress vanish in Eq. (24). The negligibility of term II supports the earlier observation on the limitation of turbulent structures to the pore size. These results can suggest that the term II is also negligible in the volume-averaged Reynolds stress presented in Eq. (17) and therefore Figs. 5, 6, 7, 8 and 9. However, since the LHS of Eq. (17) is calculated and presented in Figs. 5, 6, 7, 8 and 9, the Reynolds stress is expressed as Term II + IV in these figures. It should be noted that a 1D assumption of the flow in real porous media is relatively accurate in many applications. Khayamyan et al. $(2017 \mathrm{a}, \mathrm{b})$ showed that the most probable $y$-velocity in a randomly distributed packed bed is zero for a range of Re from laminar to turbulent flow. Therefore, only the term $V$ in Eq. (24) remains which is associated with the pore-scale spatial fluctuations of velocity 


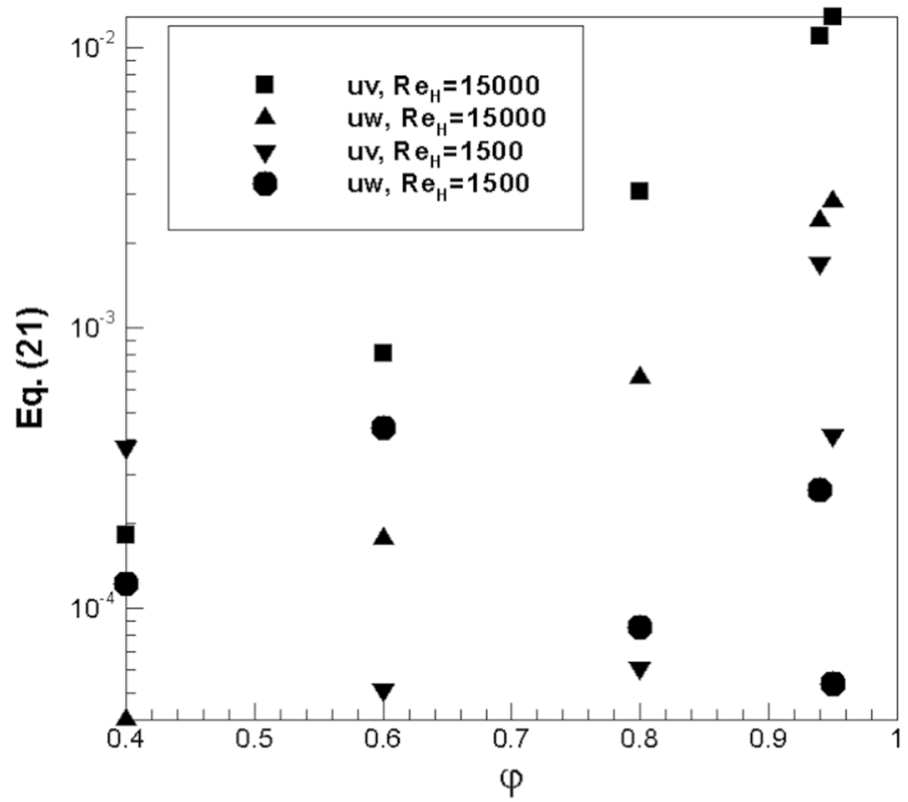

(a)

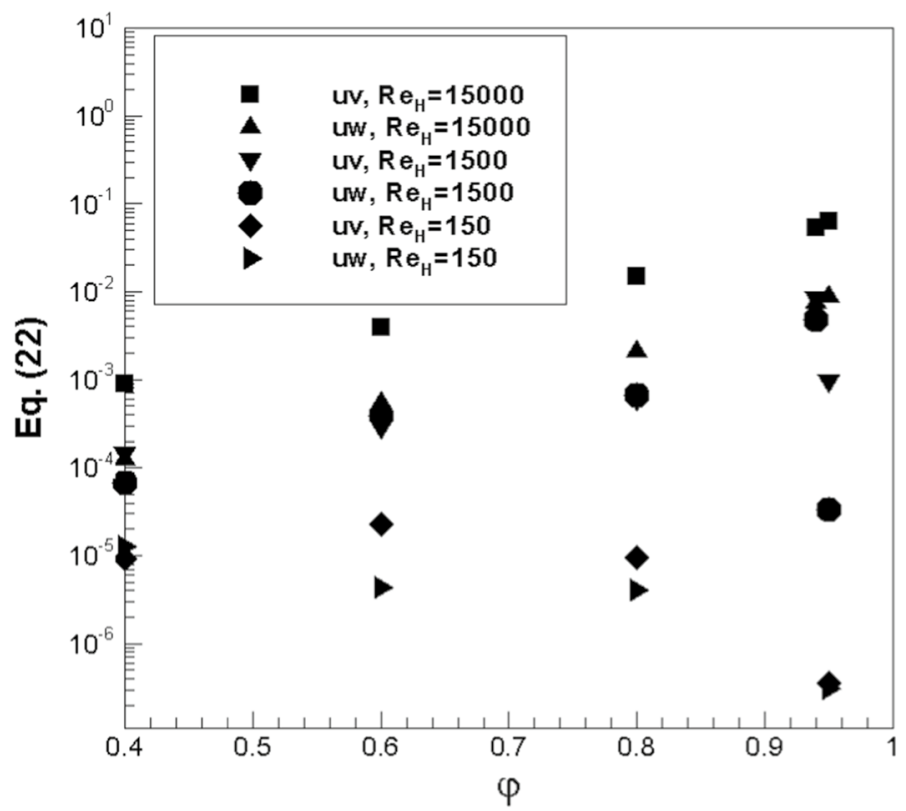

(b)

Fig. 9 Values of Eqs. (21) and (22) calculated in different porosities 
and can be decomposed into terms $I I I$ and $I V$. The aim of this section is to inspect the distribution of the term $V$ within the pores. To this end, two random contours of $\tilde{U}_{1} \tilde{U}_{2}$ are extracted for each porosity at $\operatorname{Re}_{H}=1500$, as shown in Fig. 10. A histogram of the image data has been extracted in which the range of variation in $\tilde{U}_{1} \tilde{U}_{2}$ is divided into 256 bins and the histogram counts associated with each bin are returned. Due to the white background, the histogram shows large amounts for large bins. In order to rectify this problem, the data associated with these bins are not considered to prevent the effect of this noise in the calculations. Then, the normalized probability density function (NPDF) and cumulative

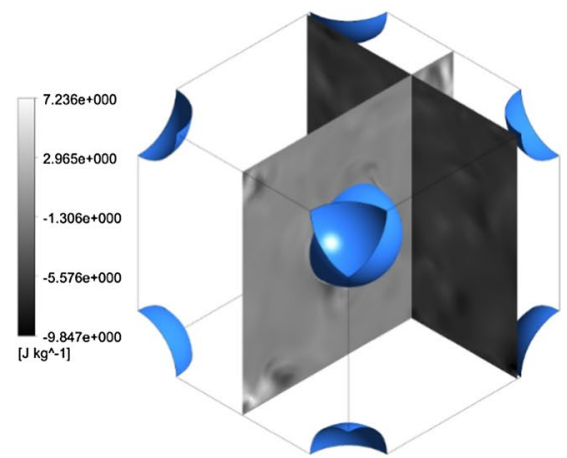

(a)

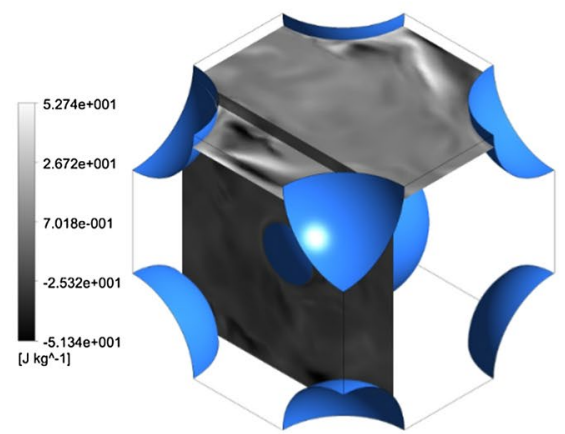

(c)

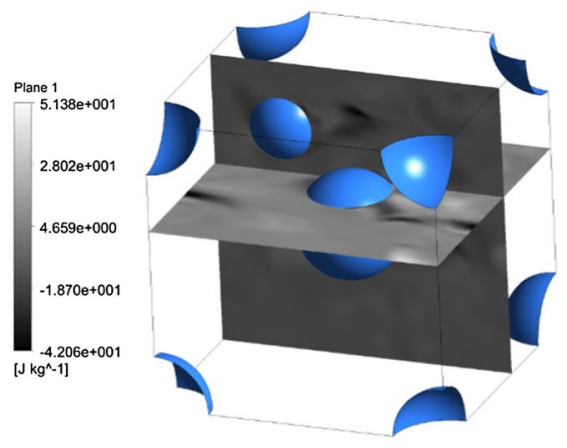

(b)

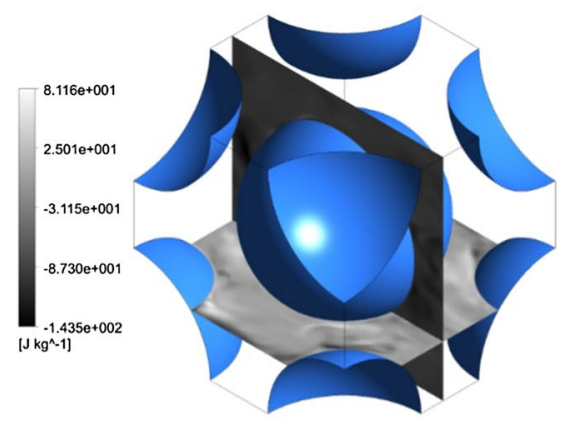

(d)

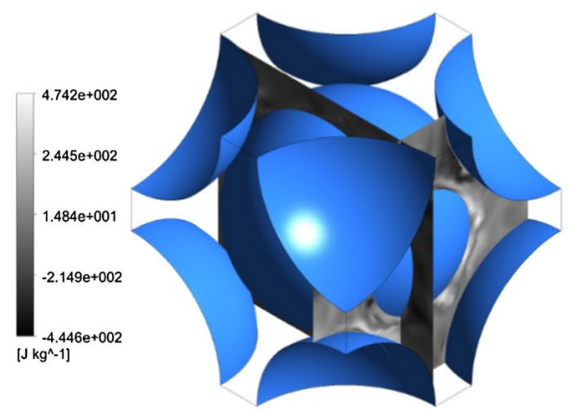

(e)

Fig. 10 Positions of two random planes in each porosity for $\mathbf{a} \varphi=0.95, \mathbf{b} \varphi=0.94, \mathbf{c} \varphi=0.8, \mathbf{d} \varphi=0.6$, e $\varphi=0.4$ 
distribution function (CDF) are used to scrutinize the $\tilde{U}_{1} \tilde{U}_{2}$ field within the planes shown in Fig. 11 . The NPDF of the $\tilde{U}_{1} \tilde{U}_{2}$ shows the probability of $\tilde{U}_{1} \tilde{U}_{2}$ values normalized with the probability of the most probable $\tilde{U}_{1} \tilde{U}_{2}$.

As can be observed in Fig. 11, the most probable values of $\tilde{U}_{1} \tilde{U}_{2}$ in two random planes are close to zero. It is also found that the CDF of this most probable value is close to the 0.5 suggesting that the probability of $\tilde{U}_{1} \tilde{U}_{2}$ values lower than this value is equal to the probability of values greater than most probable value. Therefore, the averaged value of $\tilde{U}_{1} \tilde{U}_{2}$, $\left\langle\tilde{U}_{1} \tilde{U}_{2}\right\rangle$, is negligible as observed in Figs. 5 and 6 . This observation indicates that all of the terms in Eq. (24) are negligible for the conditions considered in the present study. This is in agreement with the results in Figs. 5 and 6. It should be noted that the terms $I$ and $I I$ are not generally zero. To exemplify, if there is macroscopic turbulence and large structures in the porous medium, it is expected that term II becomes important. However, Uth et al. (2016) showed that large structures do not survive in porous media except for unrealistically high porosities such as $\varphi=0.995$.

\subsection{Reynolds Stress in the Averaged Momentum Equation}

Based on the observations made above, it is found that turbulent structures larger than the pore sizes are not present. It is also observed that turbulent structures are confined to the pores, and therefore, they are not able to affect the transport of macroscopic quantities. In this case, the validity of the eddy viscosity hypothesis is put into jeopardy as

$$
\rho \varphi\left\langle\overline{U_{i}^{\prime} U^{\prime}}{ }_{j}\right\rangle=\underbrace{\overline{\left\langle U_{i}^{\prime}\right\rangle\left\langle U_{j}^{\prime}\right\rangle}}_{I I}+\underbrace{\left\langle\overline{\tilde{U}_{i}^{\prime} \tilde{U}_{j}^{\prime}}\right\rangle}_{I V}=\mu_{t \varphi}\left[\frac{\partial\left(\varphi\left\langle\bar{U}_{i}\right\rangle\right)}{\partial x_{j}}+\frac{\partial\left(\varphi\left\langle\bar{U}_{j}\right\rangle\right)}{\partial x_{i}}\right]-\frac{2}{3} \rho \varphi\langle k\rangle \delta_{i j} .
$$

In the absence of macroscopic turbulent effects, the term $I I$ in Eq. (25) is zero meaning that microscopic turbulence structures within the pores are responsible for the macroscopic momentum diffusion which is not possible based on the conclusion made by the pore-scale simulations here. In order to further examine this conclusion, a case of turbulent flow in two unit cells located close to a solid wall is simulated for $\varphi=0.6$
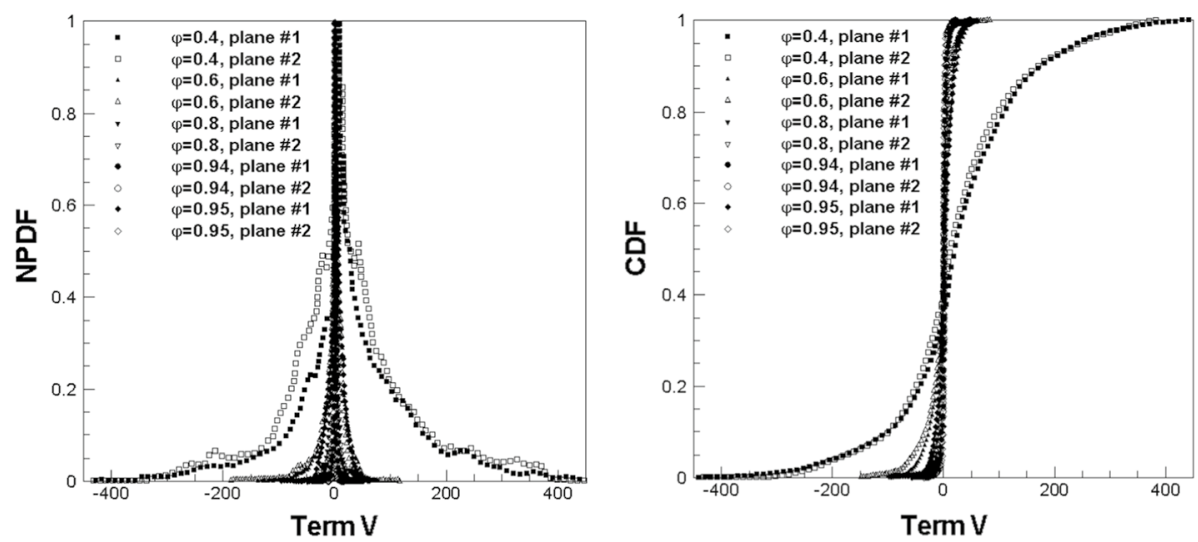

Fig. 11 Distribution of $\tilde{U}_{1} \tilde{U}_{2}$ in two random planes for different porosities 


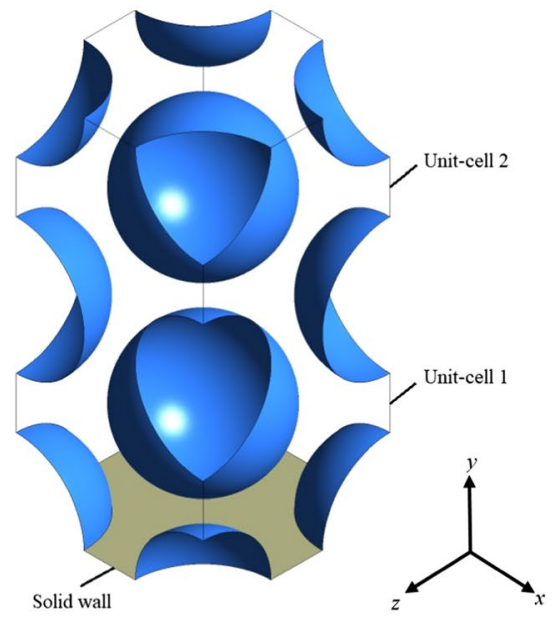

(a)

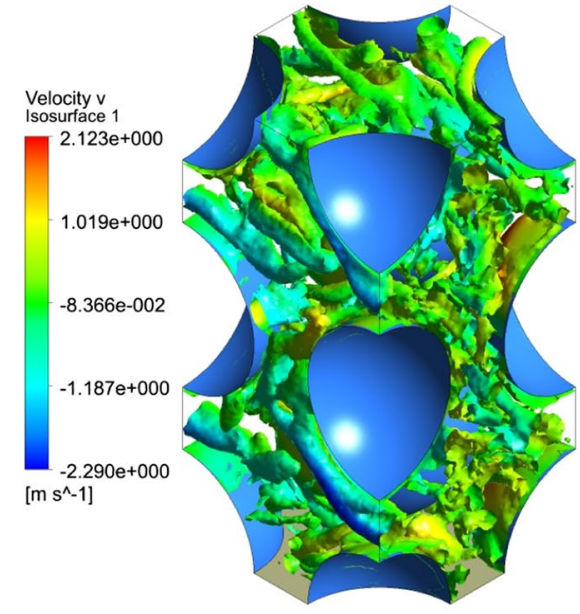

(b)

Fig. 12 a The geometry of two unit cells located close to the solid wall. b $Q$-criterion for $\varphi=0.6$, $\operatorname{Re}_{H}=1500$ and $Q / Q_{M}=0.02$

and $\operatorname{Re}_{H}=1500$, see Fig. 12a. Obviously, the macroscopic mean shear rate now is nonzero enabling an evaluation of the accuracy of the eddy viscosity hypothesis for flow in porous media. The turbulence structures in the geometry of Fig. 11a are disclosed using the iso-countors of $Q$-criterion in Fig. $12 \mathrm{~b}$. It can be observed that these structures are of the size of the pores as observed in the previous simulations. Also, the term II in Eq. (25) is negligible since $\left\langle U_{j}^{\prime}\right\rangle \approx 0$. In this case, the eddy viscosity hypothesis used in Eq. (25) incorrectly relates the volume average of the shear components of pore-scale turbulence effects, term $I V$, to macroscopic the momentum transport.

Figure 13 shows the term $I V$ as calculated from the LES and averaged over the unit cell 1 of Fig. 12a for different nondimensional times, $t^{*}$, for $i=1, j=1$ and $i=1, j=2$. It is observed that other macroscopic quantities such as normal stress, $\left\langle\overline{U^{\prime} U^{\prime}}\right\rangle / U_{\mathrm{D}}^{2}$, friction factor, $f^{\prime}$ and velocity gradient, $\frac{H}{U_{\mathrm{D}}} \frac{\partial\left(\varphi\left\langle\bar{U}_{i}\right\rangle\right)}{\partial x_{j}}$ obtain a nearly constant value after a nondimensional time of $t^{*} \approx 9 H / U_{\mathrm{D}}$, while the shear stress $\left\langle\overline{U^{\prime} V^{\prime}}\right\rangle$ continues to vary with time. This observation agrees with those presented in Figs. 5a and 6a for the volume-averaged shear stresses at different porosities. Therefore, it can be concluded that the amount of macroscopic velocity gradient is independent of the volume-averaged value of the pore-scale Reynolds stresses.

Based on the discussion presented above, it can be concluded that the intra-pore turbulence effects within the pores are not able to affect the distribution of macroscopic quantities. As a result, not only the eddy viscosity hypothesis but also all the models that present a relationship between the intra-pore turbulence and the macroscopic quantities are not accurate in this condition. 

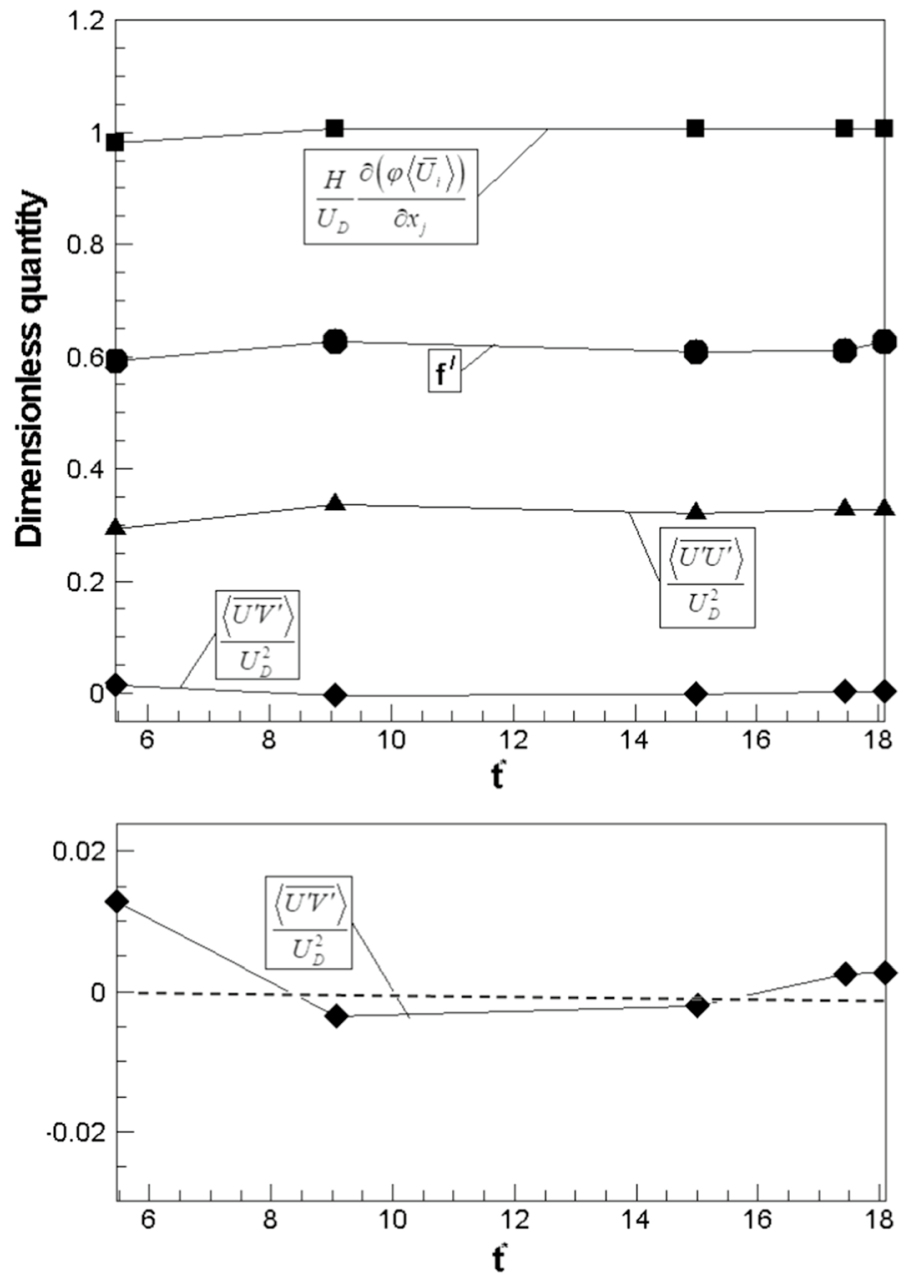

Fig. 13 Variation in macroscopic dimensionless quantities with nondimensional time

\subsection{Effect of Pore-Scale Geometry}

The results presented until now are obtained for BCC (body centered cubic system) arrangement of spheres in a unit cell and one random geometry (see Fig. 1). In this section, the effect of varying the arrangement of spherical particles to FCC (face centered cubic system) and changing the positions of 10th particle in the random geometry on the hydrodynamic dispersion and Reynolds stress components will be studied. Figure 14 illustrates the vortical structures obtained for FCC cubic cell in $\operatorname{Re}_{H}=15000$ and for $\varphi=0.6$ and $\varphi=0.95$. As can be observed, the iso-surfaces of $Q$ are similar to those found for the BCC arrangement in Fig. 3 and the vortical structures are of the order of pore size. Also, it is shown in Fig. 15 that the averaged values of hydrodynamic dispersion and Reynolds stress normal components differ slightly between BCC and FCC arrangements for $\varphi=0.8$ and $\varphi=0.95$ in $\operatorname{Re}_{H}=15000$. This can be attributed to large space between the spheres in these cases so that the change in the arrangement of spheres does not change significantly 


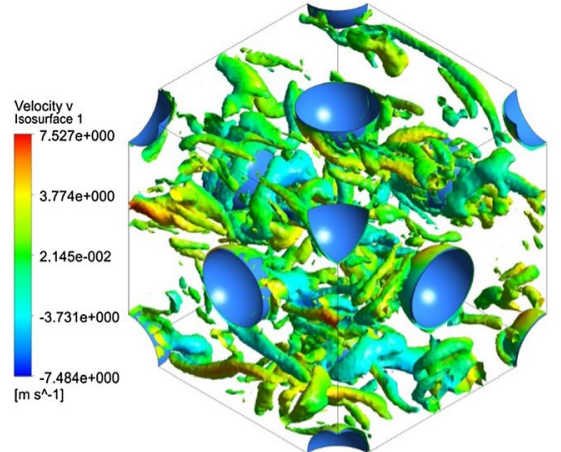

(a)

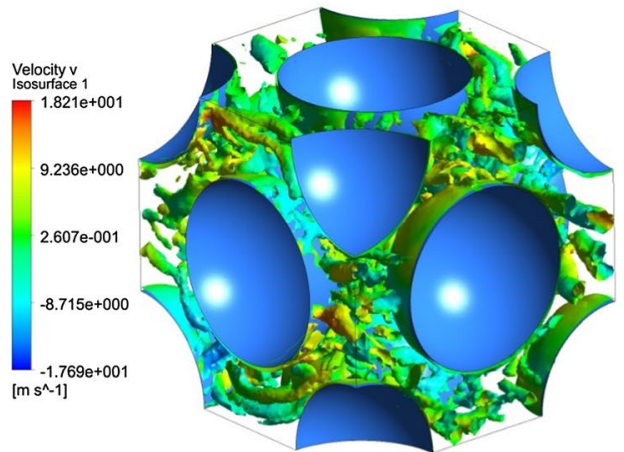

(b)

Fig. 14 Vortical structures within the unit cell identified by iso-surfaces of $Q / Q_{M}=0.02$ for $\operatorname{Re}_{H}=15000$ a $\varphi=0.95, \mathbf{b} \varphi=0.6$

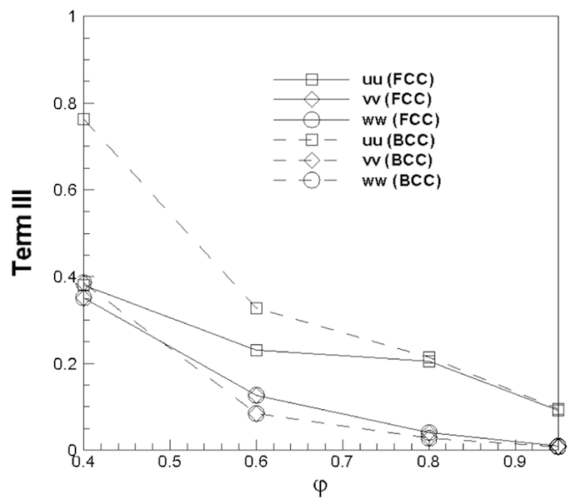

(a)

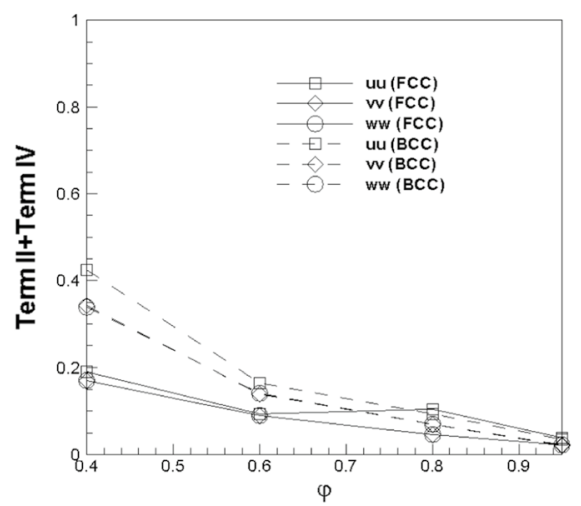

(c)

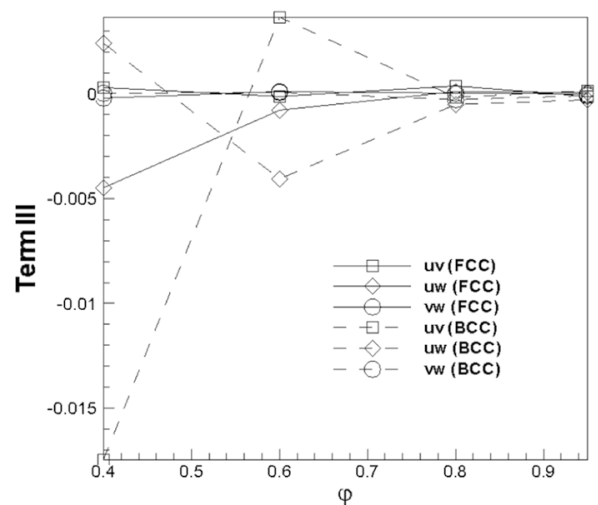

(b)

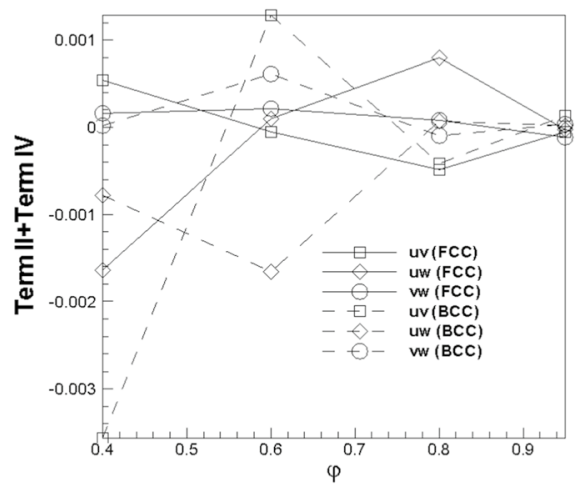

(d)

Fig. 15 Comparison of a normal components of hydrodynamic dispersion, $\mathbf{b}$ shear components of hydrodynamic dispersion, $\mathbf{c}$ normal components of Reynolds stress, $\mathbf{d}$ shear components of Reynolds stress between FCC and BCC arrangements in $\mathrm{Re}_{H}=15000$ 
the flow path and therefore these quantities. However, a somewhat different behavior is observed for $\varphi=0.4$ and $\varphi=0.6$ in Fig. 15. By putting spheres at the side surfaces of cube in FCC arrangement in lower porosities, the main flow in $x$-direction does not have enough space to disperse and is more confined to the pore space. Therefore, the normal components of hydrodynamic dispersion in $x$-direction $(u u)$ for the FCC arrangement are lower than that in BCC. Meanwhile, the level of normal components of Reynolds stresses are lower in the FCC arrangement for $\varphi=0.4$ and $\varphi=0.6$. This can be attributed to the larger surface area of solid walls in the FCC arrangement for the same spheres volume as that in BCC which damps turbulence within the flow. The shear components of hydrodynamic dispersion and Reynolds stresses in the FCC arrangement shown in Fig. 15b, $d$ have the same non-monotonic trend and range of values as those calculated in the $\mathrm{BCC}$ arrangement. Generally, the components of hydrodynamic dispersion and Reynolds stresses calculated in the BCC and FCC arrangements are of the same order of magnitude which shows that a change in the pore-scale geometry does not affect the flow structure within the pores. Figure 16 illustrates the effect of variation in the position of 10th particle in the random geometry of Fig. 1b on the intra-pore turbulence and hydrodynamic dispersion. In this case, the position of 10 th sphere is changed to $(0.166 \mathrm{H}-0.3 \mathrm{H} 0.166 \mathrm{H})$ with respect to the center of cube. It is observed that the macroscopic values of normal components of hydrodynamic dispersion and Reynolds stress are nearly similar in two cases. Although the macroscopic values of shear components vary with a change in the position of 10th sphere in the random geometry, their values are still negligible similar to those observed in Fig. 5b.

\section{Conclusion}

Pore-scale simulations of turbulent flow in the unit cells of porous media have been carried out in the present study. Through visualization of vortical structures, it is observed that the size of these structures is of the order as the pore size for all porosities and Re investigated. Therefore, it is concluded that these structures are not able to directly modify the macroscopic flow field. By averaging the hydrodynamic dispersion and Reynolds stress values over the unit cells, it is observed that they are of the same order of magnitude and their normal components increase with a decrease in porosity. Meanwhile, it is shown that the averaged values of the hydrodynamic dispersion and the Reynolds stress shear components are significantly lower than the square of the Darcian velocity which appears in the Forchheimer term. Inspecting the distribution of the hydrodynamic dispersion and intra-pore turbulence shear components in different planes for all porosities studied reveals that the most probable values of these quantities are very close to zero and their values are distributed normally in the pore space. This phenomenon is observed for all porosities regardless of ordered or non-ordered distributions of particles. Further discussion on the validity of the eddy viscosity hypothesis reveals that it is not appropriate to model the shear components of averaged intra-pore Reynolds stresses since they do not affect the macroscopic distribution of averaged quantities. Finally, the effect of variation in pore-scale geometry on the averaged dispersion and Reynolds stress values is studied and it is shown that, despite the change in values, their order of magnitudes is similar in different geometries. 


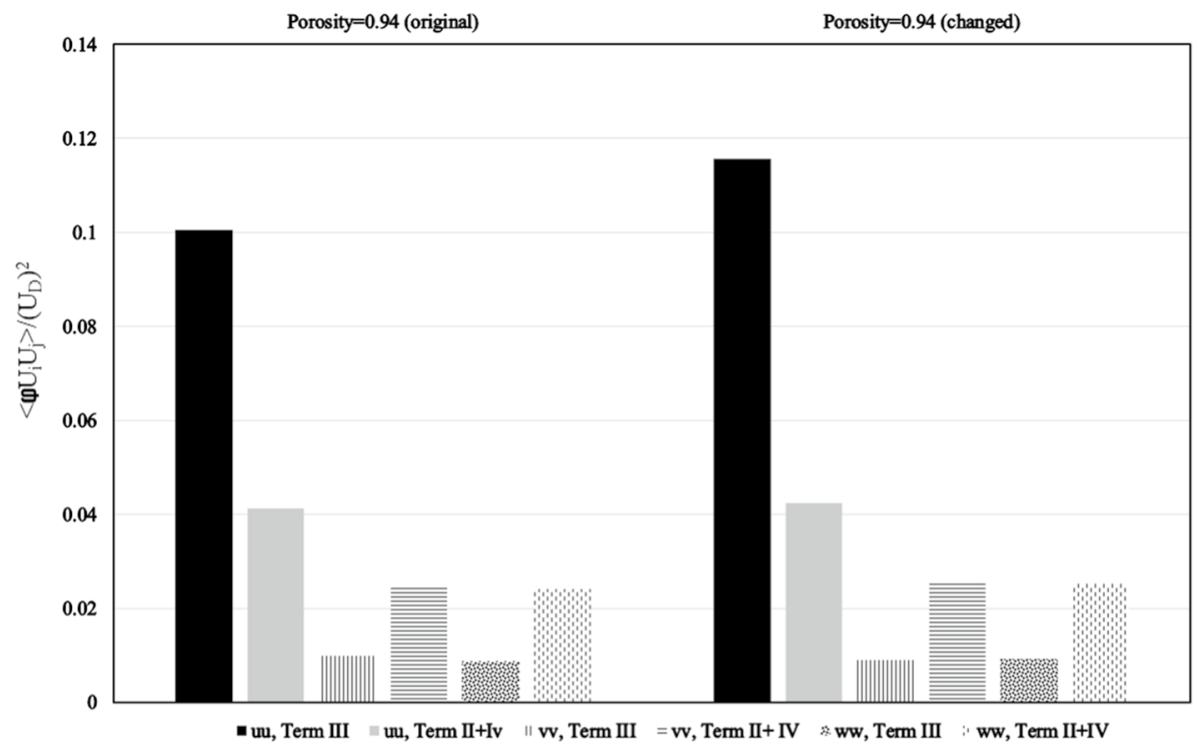

(a)

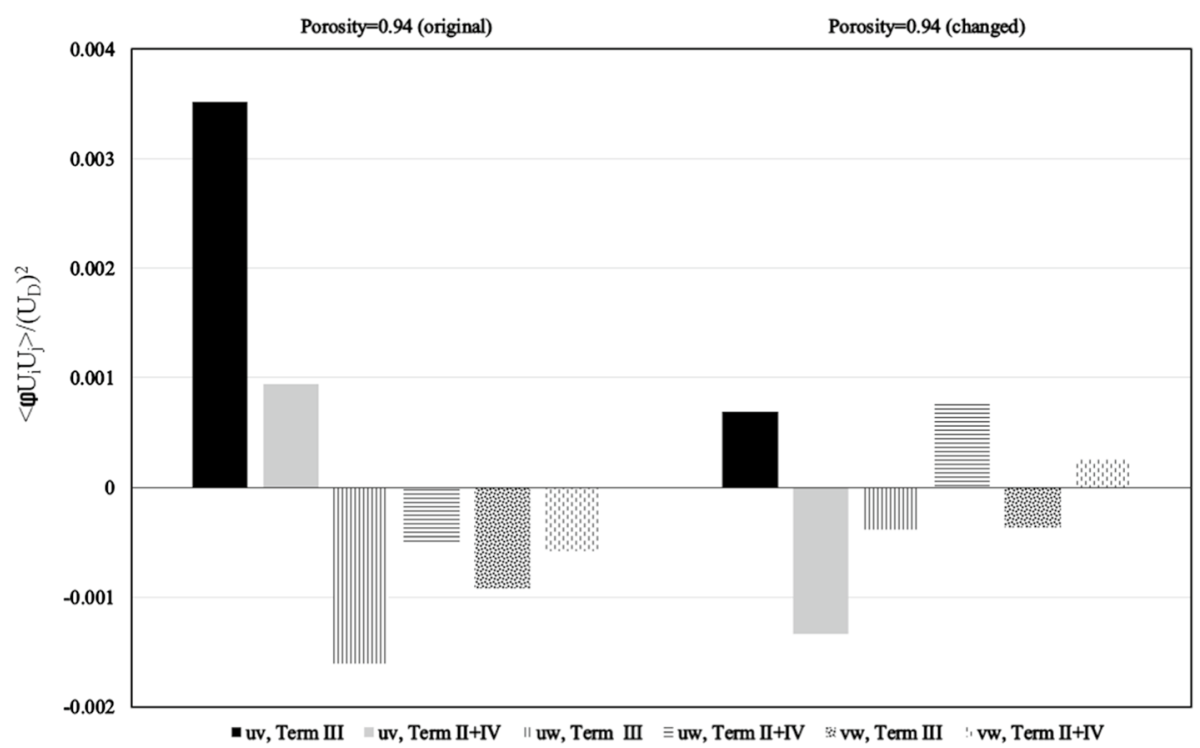

(b)

Fig. 16 The effect of varying the positions of 10th sphere on the a normal components, $\mathbf{b}$ shear components of hydrodynamic dispersion and Reynolds stress for $\operatorname{Re}_{H}=15000$ and $\varphi=0.94$

Acknowledgements Open access funding provided by Lulea University of Technology. The project was funded by the Kempe Foundations and Luleå University of Technology, and the work was carried out under the Grant 2017-04390 from the Swedish Research Council. 
Open Access This article is distributed under the terms of the Creative Commons Attribution 4.0 International License (http://creativecommons.org/licenses/by/4.0/), which permits unrestricted use, distribution, and reproduction in any medium, provided you give appropriate credit to the original author(s) and the source, provide a link to the Creative Commons license, and indicate if changes were made.

\section{References}

Alvarez, G., Bournet, P.-E., Flick, D.: Two-dimensional simulation of turbulent flow and transfer through stacked spheres. Int. J. Heat Mass Transf. 46(13), 2459-2469 (2003)

Amiri, A., Vafai, K.: Analysis of dispersion effects and non-thermal equilibrium, non-Darcian, variable porosity incompressible flow through porous media. Int. J. Heat Mass Transf. 37(6), 939-954 (1994)

Ansys, C.: User's Guide-14. ANSYS Inc., New York (2012)

Antohe, B., Lage, J.: A general two-equation macroscopic turbulence model for incompressible flow in porous media. Int. J. Heat Mass Transf. 40(13), 3013-3024 (1997)

Chu, X., Weigand, B., Vaikuntanathan, V.: Flow turbulence topology in regular porous media: from macroscopic to microscopic scale with direct numerical simulation. Phys. Fluids 30(6), 065102 (2018)

De Lemos, M.J.: Turbulent flow around fluid-porous interfaces computed with a diffusion-jump model for $k$ and $\varepsilon$ transport equations. Transp. Porous Media 78(3), 331-346 (2009)

Dybbs, A., Edwards, R.: A new look at porous media fluid mechanics-Darcy to turbulent. In: Bear, J., Corapcioglu, M.Y. (eds.) Fundamentals of Transport Phenomena in Porous Media, pp. 199-256. Springer, Dordrecht (1984)

Ergun, S., Orning, A.A.: Fluid flow through randomly packed columns and fluidized beds. Ind. Eng. Chem. 41(6), 1179-1184 (1949)

Getachew, D., Minkowycz, W., Lage, J.: A modified form of the $\kappa-\varepsilon$ model for turbulent flows of an incompressible fluid in porous media. Int. J. Heat Mass Transf. 43(16), 2909-2915 (2000)

Hellström, J., Marjavaara, B., Lundström, T.: Parallel CFD simulations of an original and redesigned hydraulic turbine draft tube. Adv. Eng. Softw. 38(5), 338-344 (2007)

Hellstrom, J.G.I., Jonsson, P.J.P., Lundstrom, S.: Laminar and turbulent flow through an array of cylinders. J. Porous Media 13(12), 1073-1085 (2010)

Jin, Y., Kuznetsov, A.: Turbulence modeling for flows in wall bounded porous media: an analysis based on direct numerical simulations. Phys. Fluids 29(4), 045102 (2017)

Jin, Y., Uth, M.-F., Kuznetsov, A., Herwig, H.: Numerical investigation of the possibility of macroscopic turbulence in porous media: a direct numerical simulation study. J. Fluid Mech. 766, 76-103 (2015)

Jouybari, N.F., Maerefat, M., Nimvari, M.E.: A macroscopic turbulence model for reacting flow in porous media. Transp. Porous Media 106(2), 355-381 (2015)

Khayamyan, S., Lundström, T.S., Gren, P., Lycksam, H., Hellström, J.G.I.: Transitional and turbulent flow in a bed of spheres as measured with stereoscopic particle image velocimetry. Transp. Porous Media 117(1), 45-67 (2017a)

Khayamyan, S., Lundström, T.S., Hellström, J.G.I., Gren, P., Lycksam, H.: Measurements of transitional and turbulent flow in a randomly packed bed of spheres with particle image velocimetry. Transp. Porous Media 116(1), 413-431 (2017b)

Kundu, P., Kumar, V., Mishra, I.: Numerical modeling of turbulent flow through isotropic porous media. Int. J. Heat Mass Transf. 75, 40-57 (2014)

Kuwahara, F., Yamane, T., Nakayama, A.: Large eddy simulation of turbulent flow in porous media. Int. Commun. Heat Mass Transf. 33(4), 411-418 (2006)

Kuwata, Y., Suga, K.: Modelling turbulence around and inside porous media based on the second moment closure. Int. J. Heat Fluid Flow 43, 35-51 (2013)

Kuwata, Y., Suga, K., Sakurai, Y.: Development and application of a multi-scale $k-\varepsilon$ model for turbulent porous medium flows. Int. J. Heat Fluid Flow 49, 135-150 (2014)

Larsson, I.S., Lundström, T.S., Lycksam, H.: Tomographic PIV of flow through ordered thin porous media. Exp. Fluids 59(6), 96 (2018)

Masuoka, T., Takatsu, Y.: Turbulence model for flow through porous media. Int. J. Heat Mass Transf. 39(13), 2803-2809 (1996)

Nakayama, A., Kuwahara, F.: A macroscopic turbulence model for flow in a porous medium. J. Fluids Eng. 121(2), 427-433 (1999)

Nakayama, A., Kuwahara, F.: A general macroscopic turbulence model for flows in packed beds, channels, pipes, and rod bundles. J. Fluids Eng. 130(10), 101205 (2008) 
Nield, D.: The limitations of the Brinkman-Forchheimer equation in modeling flow in a saturated porous medium and at an interface. Int. J. Heat Fluid Flow 12(3), 269-272 (1991)

Pedras, M.H., de Lemos, M.J.: Macroscopic turbulence modeling for incompressible flow through undeformable porous media. Int. J. Heat Mass Transf. 44(6), 1081-1093 (2001)

Pope, S.B.: Turbulent Flows. IOP Publishing, Bristol (2001)

Prahl, L., Jadoon, A., Revstedt, J.: Interaction between two spheres placed in tandem arrangement in steady and pulsating flow. Int. J. Multiph. Flow 35(10), 963-969 (2009)

Rodriguez, I., Borell, R., Lehmkuhl, O., Segarra, C.D.P., Oliva, A.: Direct numerical simulation of the flow over a sphere at $R e=3700$. J. Fluid Mech. 679, 263-287 (2011)

Rodríguez Pérez, I.M., Lehmkuhl, O., Soria Guerrero, M, Gómez, S., Domínguez Pumar, M., Kowalski, L.: On the boundary layer development and heat transfer from a sphere at moderate Reynolds numbers. In: ICCFD10 Proceedings (2018)

Seguin, D., Montillet, A., Comiti, J., Huet, F.: Experimental characterization of flow regimes in various porous media-II: transition to turbulent regime. Chem. Eng. Sci. 53(22), 3897-3909 (1998)

Sozen, M., Vafai, K.: Longitudinal heat dispersion in porous beds with real-gas flow. J. Thermophys. Heat Transfer 7(1), 153-157 (1993)

Travkin, V., Catton, I.: Models of turbulent thermal diffusivity and transfer coefficients for a regular packed bed of spheres. ASME-PUBLICATIONS-HTD 193, 15 (1992)

Uth, M.-F., Jin, Y., Kuznetsov, A., Herwig, H.: A direct numerical simulation study on the possibility of macroscopic turbulence in porous media: effects of different solid matrix geometries, solid boundaries, and two porosity scales. Phys. Fluids 28(6), 065101 (2016)

Vafai, K.: Handbook of Porous Media. CRC Press, Boca Raton (2015)

Vafai, K., Tien, C.: Boundary and inertia effects on flow and heat transfer in porous media. Int. J. Heat Mass Transf. 24(2), 195-203 (1981)

Vafai, K., Bejan, A., Minkowycz, W., Khanafer, K.: A critical systhesis of pertinent models for turbulent transport through porous media. In: Minkowycz, W.J., Sparrow, E.M., Murthy, J.Y. (eds.) Handbook of Numerical Heat Transfer, 2nd edn, Chap. 12, pp. 389-416. Wiley, Hoboken (2006)

Versteeg, H.K., Malalasekera, W.: An Introduction to Computational Fluid Dynamics: The Finite, vol. Method. Pearson education, London (2007)

Yang, J., Zhou, M., Li, S., Bu, S., Wang, Q.: Three-dimensional numerical analysis of turbulent flow in porous media formed by periodic arrays of cubic, spherical, or ellipsoidal particles. J. Fluids Eng. 136(1), $011102(2014)$

Publisher's Note Springer Nature remains neutral with regard to jurisdictional claims in published maps and institutional affiliations. 